Pacific

Journal of

Mathematics

\title{
ON THE CLASSIFICATION OF KILLING SUBMERSIONS
} AND THEIR ISOMETRIES

José M. MANZANO 


\title{
ON THE CLASSIFICATION OF KILLING SUBMERSIONS AND THEIR ISOMETRIES
}

\author{
JoSÉ M. MANZANO
}

\begin{abstract}
A Killing submersion is a Riemannian submersion from an orientable 3manifold to an orientable surface whose fibers are the integral curves of a unit Killing vector field in the 3-manifold. We classify all Killing submersions over simply connected Riemannian surfaces and give explicit models for many Killing submersions, including those over simply connected constant Gaussian curvature surfaces. We also fully describe the isometries of the total space preserving the vertical direction. As a consequence, we prove that the only simply connected homogeneous 3-manifolds which admit a structure of Killing submersion are the $\mathbb{E}(\kappa, \tau)$-spaces, whose isometry group has dimension at least 4 .
\end{abstract}

\section{Introduction}

Simply connected homogeneous Riemannian 3-manifolds with an isometry group of dimension 4 or 6 different from $\mathbb{H}^{3}$ can be represented by a 2-parameter family $\mathbb{E}(\kappa, \tau)$, where $\kappa, \tau \in \mathbb{R}$. They include $\mathbb{R}^{3}, \mathbb{S}^{3}, \mathbb{H}^{2} \times \mathbb{R}, \mathbb{S}^{2} \times \mathbb{R}$, the Heisenberg group, the Berger spheres and the universal cover of the special linear group $\mathrm{SL}_{2}(\mathbb{R})$ endowed with a left-invariant metric (see [Daniel 2007; Daniel et al. 2009; Meeks and Pérez 2012]). The $\mathbb{E}(\kappa, \tau)$-spaces are 3-manifolds admitting a global unit Killing vector field whose integral curves are the fibers of a certain Riemannian submersion over the simply connected constant Gaussian curvature surface $\mathbb{M}^{2}(\kappa)$. In the Riemannian product 3-manifolds $M \times \mathbb{R}$, the projection over the first factor is a Riemannian submersion whose fibers are also the trajectories of a unit Killing vector field. In general, Riemannian submersions sharing this property will be called Killing submersions (see [Espinar and de Oliveira 2013; Rosenberg et al. 2010] and Definition 1.1 below).

Constant mean curvature surfaces in $\mathbb{E}(\kappa, \tau)$ and $M \times \mathbb{R}$ have been extensively studied during the last decade and many results have been recently extended to the

Research partially supported by the Spanish MCI research projects MTM2007-61775 and MTM201122547, and the Junta de Andalucía Grant P09-FQM-5088.

MSC2010: primary 53C15; secondary 53C 30.

Keywords: unit Killing vector field, Riemannian submersions, homogeneous spaces. 
Killing submersion setting (e.g., see [Dajczer and de Lira 2009, 2012; Espinar and de Oliveira 2013; Leandro and Rosenberg 2009; Rosenberg et al. 2010; Meroño and Ortiz 2014]). Nevertheless, apart from the aforementioned spaces, the theory of Killing submersions suffers from a lack of examples. It is necessary to mention that these 3-manifolds are well-understood at the level of differential topology (see [Besse 2008; Greub et al. 1976; Steenrod 1951]) since the projection defines principal bundles with totally geodesic fibers. Nevertheless, the objective of this paper is to classify them in the Riemannian category provided that the base is simply connected, and give explicit models depending on the base surface and a special geometric function, the so-called bundle curvature.

The bundle curvature has proved to be a very natural function in the surface theory of Killing submersions. For instance, a Calabi-type correspondence for surfaces which are graphs in the direction of the unit Killing field has been obtained recently [Lee and Manzano 2013], swapping the bundle curvature and the mean curvature of the graph. Note that Killing submersions also have dual Lorentzian counterparts when the Killing vector field is assumed to be timelike: they lead to interesting stationary spacetimes and are also related to Finsler metrics (see [Javaloyes et al. 2013]).

Let $\pi: \mathbb{E} \rightarrow M$ be a differentiable submersion from a Riemannian 3-manifold $\mathbb{E}$ onto a surface $M$. A vector $v \in T \mathbb{E}$ will be called vertical when $v \in \operatorname{ker}(\mathrm{d} \pi)$ and horizontal when $v \in \operatorname{ker}(\mathrm{d} \pi)^{\perp}$. The submersion $\pi$ is Riemannian when it preserves the length of horizontal vectors.

Definition 1.1. The Riemannian submersion $\pi: \mathbb{E} \rightarrow M$, where $\mathbb{E}$ and $M$ are connected and orientable, is called a Killing submersion if it admits a complete vertical unit Killing vector field.

As a matter of fact, any 3-manifold $\bar{M}$ admitting a unit Killing vector field $\xi$ is locally isometric to the total space of a certain Killing submersion, so the definition is not as restrictive as it may seem.

The bundle curvature of a Killing submersion $\pi: \mathbb{E} \rightarrow M$ is defined (see Lemma 2.1) as the unique function $\tau \in \mathscr{C}^{\infty}(\mathbb{E})$ satisfying

$$
\bar{\nabla}_{X} \xi=\tau X \wedge \xi \text { for all } X \in \mathfrak{X}(\mathbb{E}),
$$

where $\wedge$ is the cross product in $\mathbb{E}, \xi$ is a vertical unit Killing vector field, and $\bar{\nabla}$ denotes the Levi-Civita connection in $\mathbb{E}$. The bundle curvature is constant along the fibers of $\pi$ so it can be seen as a function $\tau \in \mathscr{C}^{\infty}(M)$ (see Propositions 3.3 and 4.6 for other geometric interpretations of $\tau$ ). This gives rise to some natural questions: Given a Riemannian surface $M$ and $\tau \in \mathscr{C}^{\infty}(M)$, does there exist a Killing submersion over $M$ with bundle curvature $\tau$ ? Is it unique? The main aim of Sections 2 and 4 will be to give affirmative answers to these questions when $M$ 
is simply connected. More specifically, we will classify Killing submersions up to isomorphism, in the following sense:

Definition 1.2. Let $\pi: \mathbb{E} \rightarrow M$ and $\pi^{\prime}: \mathbb{E}^{\prime} \rightarrow M^{\prime}$ be two Killing submersions. A (local) isomorphism of Killing submersions from $\pi$ to $\pi^{\prime}$ is a pair $(f, h)$, where $h: M \rightarrow M^{\prime}$ is an isometry and $f: \mathbb{E} \rightarrow \mathbb{E}^{\prime}$ is a (local) isometry, such that $\pi^{\prime} \circ f=h \circ \pi$.

Note that if $(f, h)$ is an isomorphism of Killing submersions, then $f$ maps fibers of $\pi$ into fibers of $\pi^{\prime}$, and, if we consider a unit vertical Killing vector field $\xi$ in $\mathbb{E}$, then $f_{*} \xi$ is also a unit vertical Killing vector field in $\mathbb{E}^{\prime}$.

Given a simply connected Riemannian surface $M$ and $\tau \in \mathscr{C}^{\infty}(M)$, we will show that there exists a Killing submersion over $M$ with bundle curvature $\tau$, and it is unique (up to isomorphism) if the total space $\mathbb{E}$ is also simply connected. In the process, it will turn out that the bundle curvature determines locally the geometry of the submersion, but the topology of $\mathbb{E}$ is also conditioned by the bundle curvature. More explicitly:

- If $M$ is a topological disk, then the submersion is isomorphic to the projection $\pi_{1}: M \times \mathbb{R} \rightarrow M, \pi_{1}(p, t)=p$, for some Riemannian metric on $M \times \mathbb{R}$ such that $\partial_{t}$ is a unit vertical Killing vector field. In particular, the fibers of the submersion have infinite length.

- If, on the contrary, $M=\left(\mathbb{S}^{2}, g\right)$ for some Riemannian metric $g$, then we shall distinguish cases depending on whether the total bundle curvature $T=\int_{M} \tau$ vanishes or not:

- If $T=0$, then $\pi$ is isomorphic to $\pi_{1}: \mathbb{S}^{2} \times \mathbb{R} \rightarrow\left(\mathbb{S}^{2}, g\right), \pi_{1}(p, t)=p$, for some metric on $\mathbb{S}^{2} \times \mathbb{R}$ such that $\partial_{t}$ is a unit vertical Killing vector field, so the fibers have infinite length.

- If $T \neq 0$, then $\pi$ is isomorphic to $\pi_{\text {Hopf }}: \mathbb{S}^{3} \rightarrow\left(\mathbb{S}^{2}, g\right), \pi_{\mathrm{Hopf}}(z, w)=$ $\left(2 z \bar{w},|z|^{2}-|w|^{2}\right)$, where $\mathbb{S}^{3} \subset \mathbb{C}^{2}$ is endowed with a metric such that $(\pi / T)(i z, i w)$ is a unit vertical Killing vector field. In this case, the fibers have length $|2 T|$.

When the total space is not simply connected, Killing submersions over $M$ are also classified as the quotients of those listed above under a vertical translation (i.e., an element of the 1-parameter group of isometries associated to the unit Killing vector field).

Though this theoretical description is exhaustive, we will give explicit models for a wide class of Killing submersions. Firstly, for those over a disk with a conformal metric in terms of the conformal factor, the obtained examples will generalize the metrics for the $\mathbb{E}(\kappa, \tau)$-spaces in [Daniel 2007]. Secondly, we will obtain a general method to produce trivial Killing submersions (i.e., admitting a global smooth 
section) over any surface by isometrically embedding it in $\mathbb{R}^{n}$ for some $n \geq 3$. Finally, explicit models will also be obtained for Killing submersions over the round sphere $\mathbb{S}^{2}(\kappa)$ via the Hopf fibration (generalizing the metrics of the Berger spheres in [Torralbo 2012]).

The geometries of $M$ and $\mathbb{E}$ of a Killing submersion $\pi: \mathbb{E} \rightarrow M$ are well-related, and geodesics or isometries are good samples of that. On the one hand, geodesics of $\mathbb{E}$ can be divided into three different types: vertical ones, horizontal ones (which are horizontal lifts of geodesics of $M$ ) and those which are neither vertical nor horizontal, each of which makes a constant angle with the vertical direction and whose projection is well-understood (see Proposition 3.6). In particular, $M$ is complete if and only if $\mathbb{E}$ is complete. On the other hand, a beautiful classification result is obtained when we look for Killing isometries (i.e., isometries of $\mathbb{E}$ preserving the vertical direction). More explicitly, if $\mathbb{E}$ and $M$ are simply connected, and $\tau \in \mathscr{C}^{\infty}(M)$ denotes the bundle curvature, then:

(a) Given a Killing isometry $f: \mathbb{E} \rightarrow \mathbb{E}$, there exists a unique isometry $h: M \rightarrow M$ such that $\pi \circ f=h \circ \pi$. Moreover, $\tau \circ h=\tau$ if $f$ is orientation-preserving and $\tau \circ h=-\tau$ if it is orientation-reversing.

(b) Conversely, given an isometry $h: M \rightarrow M$ and $p_{0}, q_{0} \in \mathbb{E}$ with $h\left(\pi\left(p_{0}\right)\right)=$ $\pi\left(q_{0}\right)$, the following properties hold:

- If $\tau \circ h=\tau$, then there is a unique orientation-preserving Killing isometry $f: \mathbb{E} \rightarrow \mathbb{E}$ with $\pi \circ f=h \circ \pi$ and $f\left(p_{0}\right)=q_{0}$.

- If $\tau \circ h=-\tau$, then there is a unique orientation-reversing Killing isometry $f: \mathbb{E} \rightarrow \mathbb{E}$ with $\pi \circ f=h \circ \pi$ and $f\left(p_{0}\right)=q_{0}$.

This construction provides a surjective group morphism from the group of Killing isometries of $\mathbb{E}$ to the group of isometries of $M$ which either preserve $\tau$ or map it to $-\tau$. Its kernel consists of isometries of $\mathbb{E}$ that leave the fibers invariant (i.e., vertical translations, and also symmetries with respect to a horizontal slice when $\tau=0$ ). In particular, 1-parameter groups of isometries of $M$ preserving $\tau$ give rise to 1-parameter groups of isometries in $\mathbb{E}$. Such groups have proven to be essential in surface theory, leading to many geometric features, e.g., they are related to holomorphic quadratic differentials (see [Abresch and Rosenberg 2005]) and conjugate constructions (see [Manzano and Torralbo 2012]).

Finally, note that simply connected homogeneous 3-manifolds are classified: they are all isometric to Lie groups endowed with left-invariant metrics except for $\mathbb{S}^{2}(\kappa) \times \mathbb{R}$, where $\kappa>0$ (see [Meeks and Pérez 2012, Theorem 2.4]). In Section 5, we will characterize the homogeneous spaces $\mathbb{E}(\kappa, \tau)$ as the only simply connected homogeneous 3-manifolds admitting a Killing submersion structure (see Theorem 5.2). Hence, the only Killing submersions whose total space is isometric to a Lie group endowed with a left invariant metric are the $\mathbb{E}(\kappa, \tau)$-spaces, except for $\mathbb{S}^{2}(\kappa) \times \mathbb{R}$. 


\section{Uniqueness results}

The bundle curvature. The next result can be found in [Espinar and de Oliveira 2013; Souam and Van der Veken 2012], but we will include the proof here for completeness.

Lemma 2.1. Let $\pi: \mathbb{E} \rightarrow M$ be a Killing submersion. Then there exists a function $\tau \in \mathscr{C}^{\infty}(\mathbb{E})$ such that $\bar{\nabla}_{X} \xi=\tau X \wedge \xi$ for all $X \in \mathfrak{X}(\mathbb{E})$.

The function $\tau$ will be called the bundle curvature of the submersion.

Proof. First of all, note that $\bar{\nabla}_{\xi} \xi=0$. Indeed, given $X \in \mathfrak{X}(\mathbb{E})$, we have

$$
\left\langle\bar{\nabla}_{\xi} \xi, X\right\rangle=-\left\langle\bar{\nabla}_{X} \xi, \xi\right\rangle=-\frac{1}{2} X\langle\xi, \xi\rangle=0,
$$

since $\xi$ is Killing and unitary.

Let us now take $X \in \mathfrak{X}(\mathbb{E})$ linearly independent of $\xi$. On the one hand, it is clear that $\left\langle\bar{\nabla}_{X} \xi, \xi\right\rangle=0$ and, on the other hand, $\left\langle\bar{\nabla}_{X} \xi, X\right\rangle=0$ since $\xi$ is Killing. Then there exists a unique function $\tau_{X} \in \mathscr{C}^{\infty}(\mathbb{E})$ such that $\bar{\nabla}_{X} \xi=\tau_{X} X \wedge \xi$, so it suffices to prove that $\tau_{X}$ does not depend on $X$. It is clear that $\tau_{X}$ only depends on the horizontal part of $X$ so it will be enough to prove that $\tau_{X}=\tau_{Y}$ for all $X, Y \in \mathfrak{X}(\mathbb{E})$ horizontal. By using again that $\xi$ is a Killing vector field, we get

$$
\tau_{Y}\langle Y \wedge \xi, X\rangle=\left\langle\bar{\nabla}_{Y} \xi, X\right\rangle=-\left\langle\bar{\nabla}_{X} \xi, Y\right\rangle=-\tau_{X}\langle X \wedge \xi, Y\rangle=\tau_{X}\langle Y \wedge \xi, X\rangle,
$$

so $\tau_{X}=\tau_{Y}$, where $X$ and $Y$ are linearly independent. Elsewhere, the identity $\tau_{X}=\tau_{Y}$ follows from the linearity of the connection.

Observe that the function $\tau$ in the conditions of Lemma 2.1 is unique and its sign depends on the choice of orientation in $\mathbb{E}$. We will give now some consequences of this result in order to fix some notation.

Remark 2.2. (1) The condition $\bar{\nabla}_{\xi} \xi=0$ implies that the fibers of the submersion are geodesics of $\mathbb{E}$, which will be called vertical geodesics.

(2) The elements of the 1-parameter group of isometries $\left\{\phi_{t}\right\}_{t \in \mathbb{R}}$ associated to the Killing vector field $\xi$ will be called vertical translations.

Note that $\phi_{t}$ preserves the Killing field $\xi$ and the orientation in $\mathbb{E}$. Thus, if we apply $d \phi_{t}$ to the identity in Lemma 2.1, we easily get $\tau=\tau \circ \phi_{t}$ for all $t \in \mathbb{R}$. This means that the bundle curvature is constant along the fibers and, hence, it may be considered as a function either in $\mathbb{E}$ or in the base $M$.

(3) More generally, let $(f, h)$ be an isomorphism between two Killing submersions $\pi: \mathbb{E} \rightarrow M$ and $\pi^{\prime}: \mathbb{E}^{\prime} \rightarrow M^{\prime}$ (see Definition 1.2) and define $\tau \in \mathscr{C}^{\infty}(\mathbb{E})$ and $\tau^{\prime} \in \mathscr{C}^{\infty}\left(\mathbb{E}^{\prime}\right)$ as their bundle curvatures with respect to some orientations in $\mathbb{E}$ and $\mathbb{E}^{\prime}$, respectively. Then $\tau \circ f=\tau$ when $f$ preserves the orientation, and $\tau \circ f=-\tau^{\prime}$ when $f$ reverses the orientation. 
In the product spaces $M \times \mathbb{R}$ the projection over the first factor is a Killing submersion, so its bundle curvature is $\tau \equiv 0$ (from Lemma 2.1 it is easy to deduce that $\tau \equiv 0$ in a Killing submersion if and only if the horizontal distribution in the total space is integrable). Given $\kappa, \tau \in \mathbb{R}$, there exists a Killing submersion $\pi: \mathbb{E}(\kappa, \tau) \rightarrow \mathbb{M}^{2}(\kappa)$ with constant bundle curvature $\tau$. If $\kappa>0$ and $\tau \neq 0$, the projection is the Hopf fibration and we obtain the Berger spheres; in the remaining cases the fibers have infinite length. We refer the reader to [Daniel 2007] for a description of these examples, although Berger spheres from a global point of view can be found in [Torralbo 2012].

Other examples derived from the aforementioned ones are their Riemannian quotients by a convenient vertical translation. Thus the length of the fibers will play an important role in the theory. Since fibers are geodesics, the following result follows from [Besse 2008, Theorem 9.56].

Lemma 2.3. Let $\pi: \mathbb{E} \rightarrow M$ be a Killing submersion. Then all the fibers of $\pi$ share the same (finite or infinite) length.

Local representation of a Killing submersion. Given a surface $M$ and $\tau \in \mathscr{C}^{\infty}(M)$, we are interested in finding all Killing submersions over $M$ with bundle curvature $\tau$. Let us begin by giving a useful technical tool that will simplify some arguments throughout the paper.

Proposition 2.4. Let $\pi: \mathbb{E} \rightarrow M$ be a Killing submersion, and suppose that $M$ is noncompact. Then $\pi$ admits a global smooth section $F: M \rightarrow \mathbb{E}$. Hence,

$$
\Psi: M \times \mathbb{R} \rightarrow \mathbb{E}, \quad \Psi(p, t)=\phi_{t}(F(p)),
$$

is a local diffeomorphism, where $\left\{\phi_{t}\right\}$ denotes the 1-parameter group of vertical translations. Moreover, $\Psi$ is a global diffeomorphism if and only if the fibers of $\pi$ have infinite length.

Proof. We can suppose that the fibers of $\pi$ have finite length (otherwise, we take a quotient of $\pi$ under a vertical translation $\phi_{t}$ for some $t>0$ ). Then $\pi$ is a codimension-one circle bundle over a noncompact surface and [Greub et al. 1976, Section VIII.5] yields the existence of a global smooth section. Moreover, $\Psi$ is a local diffeomorphism since its differential is injective at every point.

Finally, note that $\Psi$ is a global diffeomorphism if and only if it is injective, but $\Psi\left(p^{\prime}, t^{\prime}\right)=\Psi(p, t)$ implies $p=p^{\prime}$ since $\Psi\left(p^{\prime}, t^{\prime}\right)$ and $\Psi(p, t)$ belong to the same fiber of $\pi$, so the last assertion in the statement holds.

This result will be mostly used to ensure that there exists a smooth section $F: U \rightarrow \mathbb{E}$ for any coordinate chart $(U, \varphi)$ in $M$, but it also implies that exceptional topologies for the total space may only arise when the base is compact. Note that, 
if the base is compact, then Proposition 2.4 no longer holds, as the Hopf fibration from $\mathbb{S}^{3}$ to $\mathbb{S}^{2}$ shows.

The following result will be the cornerstone of the subsequent development yielding a standard way of describing $\pi$ in terms of $M$ and $\tau$.

Proposition 2.5. Let $\pi: \mathbb{E} \rightarrow M$ be a Killing submersion. Let $U \subset M$ be an open set such that there is a conformal diffeomorphism $\varphi: U \rightarrow \Omega \subset \mathbb{R}^{2}$. Then:

(a) Given a smooth section $F_{0}: U \rightarrow \pi^{-1}(U)$, the transformation

$$
f: \Omega \times \mathbb{R} \rightarrow \pi^{-1}(U), \quad(x, y, t) \mapsto \phi_{t}\left(F_{0}\left(\varphi^{-1}(x, y)\right),\right.
$$

is a local diffeomorphism and satisfies $\pi \circ f=\varphi \circ \pi_{1}$ in $\Omega \times \mathbb{R}$, where $\pi_{1}: \Omega \times \mathbb{R} \rightarrow \Omega$ is the projection over the first factor.

(b) Let us write the induced metric in $\Omega$ as $\mathrm{d} s_{\lambda}^{2}=\lambda^{2}\left(\mathrm{~d} x^{2}+\mathrm{d} y^{2}\right)$ for some positive $\lambda \in \mathscr{C}^{\infty}(\Omega)$. Then there exist $a, b \in \mathscr{C}^{\infty}(\Omega)$ such that the metric in $\Omega \times \mathbb{R}$ which makes $f$ a local isometry can be expressed as

$$
\mathrm{d} s^{2}=\lambda^{2}\left(\mathrm{~d} x^{2}+\mathrm{d} y^{2}\right)+(\mathrm{d} t-\lambda(a \mathrm{~d} x+b \mathrm{~d} y))^{2} .
$$

(c) $\pi_{1}:\left(\Omega \times \mathbb{R}, \mathrm{d} s^{2}\right) \rightarrow\left(\Omega, \mathrm{d} s_{\lambda}^{2}\right)$ is a Killing submersion with unit Killing vector field $\partial_{t}$, and $\left(f, \varphi^{-1}\right)$ is a local isomorphism from $\pi_{1}$ to $\pi$.

Moreover, if the fibers of $\pi$ have infinite length, then $f$ is a global diffeomorphism. Proof. We deduce from Proposition 2.4 that $\Psi: U \times \mathbb{R} \rightarrow \pi^{-1}(U)$ given by $\Psi(p, t)=\phi_{t}\left(F_{0}(p)\right)$ is a local diffeomorphism, so $f=\Psi \circ\left(\varphi^{-1} \times \operatorname{id}_{\mathbb{R}}\right)$ is also a local diffeomorphism, and it obviously satisfies the condition $\varphi \circ \pi_{1}=\pi \circ f$, so (a) is proved. Note that Proposition 2.4 also ensures that $f$ is a global diffeomorphism if the fibers of $\pi$ have infinite length.

To prove (b), consider the unique Riemannian metric $\mathrm{d} s^{2}$ in $\varphi(U) \times \mathbb{R}$ making $f$ a local isometry. The condition $\varphi \circ \pi_{1}=\pi \circ f$ implies that $\pi_{1}$ is a Killing submersion. Vertical translations for $\pi$ correspond (through $f$ ) to isometries of the form $(x, y, t) \mapsto(x, y, t+\mu), \mu \in \mathbb{R}$, in $\left(\varphi(U) \times \mathbb{R}, \mathrm{d} s^{2}\right)$. In particular, $\partial_{t}$ is a unit vertical Killing vector field in $\left(\varphi(U) \times \mathbb{R}, \mathrm{d} s^{2}\right)$.

Let $\left\{e_{1}, e_{2}\right\}$ be the orthonormal frame in $\left(\varphi(U), \mathrm{d} s_{\lambda}^{2}\right)$, where $e_{1}=(1 / \lambda) \partial_{x}$ and $e_{2}=(1 / \lambda) \partial_{y}$, and let $\left\{E_{1}, E_{2}\right\}$ be the horizontal lift of $\left\{e_{1}, e_{2}\right\}$ with respect to $\pi_{1}$ and $E_{3}=\partial_{t}$. Since $\pi_{1}$ is the projection over the first two variables, there exist $a, b \in \mathscr{C}^{\infty}(\varphi(U))$ such that

$$
\left\{\begin{array}{l}
\left(E_{1}\right)_{(x, y, t)}=\frac{1}{\lambda(x, y)} \partial_{x}+a(x, y) \partial_{t}, \\
\left(E_{2}\right)_{(x, y, t)}=\frac{1}{\lambda(x, y)} \partial_{y}+b(x, y) \partial_{t}, \\
\left(E_{3}\right)_{(x, y, t)}=\partial_{t} .
\end{array}\right.
$$


Note that $\left\{E_{1}, E_{2}, E_{3}\right\}$ is an orthonormal frame in $\left(\varphi(U) \times \mathbb{R}, \mathrm{d} s^{2}\right)$ which can be supposed positively oriented after possibly swapping $e_{1}$ and $e_{2}$. Now it is straightforward to show that the global frame (2-3) is orthonormal for $\mathrm{d} s^{2}$ if and only if $\mathrm{d} s^{2}$ is the metric given by (2-2).

Regardless of the values of the functions $a, b \in \mathscr{C}^{\infty}(\Omega)$, the Riemannian metric given by (2-2) has the property that the projection over the first two variables is a Killing submersion over $\left(\Omega, \mathrm{d} s_{\lambda}^{2}\right)$.

Definition 2.6 (canonical example). Given an open set $\Omega \subset \mathbb{R}^{2}$ and $\lambda, a, b \in \mathscr{C}^{\infty}(\Omega)$ with $\lambda>0$, the Killing submersion

$$
\begin{gathered}
\pi_{1}:\left(\Omega \times \mathbb{R}, \mathrm{d} s_{\lambda, a, b}^{2}\right) \mapsto\left(\Omega, \mathrm{d} s_{\lambda}^{2}\right), \quad \pi_{1}(x, y, z)=(x, y), \\
\mathrm{d} s_{\lambda, a, b}^{2}=\lambda^{2}\left(\mathrm{~d} x^{2}+\mathrm{d} y^{2}\right)+(\mathrm{d} z-\lambda(a \mathrm{~d} x+b \mathrm{~d} y))^{2},
\end{gathered}
$$

will be called the canonical example associated to $(\lambda, a, b)$.

Equation (2-3) defines a global orthonormal frame $\left\{E_{1}, E_{2}, E_{3}\right\}$ for $\mathrm{d} s_{\lambda, a, b}^{2}$, where $E_{1}$ and $E_{2}$ are horizontal, and $E_{3}$ is a unit vertical Killing field. It is easy to check that $\left[E_{1}, E_{3}\right]=\left[E_{2}, E_{3}\right]=0$ and

$$
\left[E_{1}, E_{2}\right]=\frac{\lambda y}{\lambda^{2}} E_{1}-\frac{\lambda_{x}}{\lambda^{2}} E_{2}+\left(\frac{1}{\lambda^{2}}\left(b \lambda_{x}-a \lambda_{y}\right)+\frac{1}{\lambda}\left(b_{x}-a_{y}\right)\right) E_{3} .
$$

Taking into account Lemma 2.1, we can compute the bundle curvature $\tau$ associated to this canonical example as

$$
\begin{aligned}
2 \tau & =\left\langle\bar{\nabla}_{E_{1}} E_{2}, E_{3}\right\rangle-\left\langle\bar{\nabla}_{E_{2}} E_{1}, E_{3}\right\rangle=\left\langle\left[E_{1}, E_{2}\right], E_{3}\right\rangle \\
& =\frac{1}{\lambda^{2}}\left(b \lambda_{x}-a \lambda_{y}\right)+\frac{1}{\lambda}\left(b_{x}-a_{y}\right)=\frac{1}{\lambda^{2}}\left((\lambda b)_{x}-(\lambda a)_{y}\right) .
\end{aligned}
$$

This divergence formula will come in handy in the sequel.

Lemma 2.7 (classification of canonical examples). Let $\Omega \subseteq \mathbb{R}^{2}$ be a simply connected open set and $\lambda, a_{0}, a_{1}, b_{0}, b_{1} \in \mathscr{C}^{\infty}(\Omega)$ such that $\lambda>0$. The following assertions are equivalent:

(i) There exists $d \in \mathscr{C}^{\infty}(\Omega)$ such that the pair $\left(f_{d}, \operatorname{id}_{\Omega}\right)$, where

$$
\begin{aligned}
f_{d}:\left(\Omega \times \mathbb{R}, \mathrm{d} s_{\lambda, a_{0}, b_{0}}^{2}\right) & \rightarrow\left(\Omega \times \mathbb{R}, \mathrm{d} s_{\lambda, a_{1}, b_{1}}^{2}\right), \\
(x, y, z) & \mapsto(x, y, z-d(x, y)),
\end{aligned}
$$

is an isomorphism of Killing submersions.

(ii) There exists $d \in \mathscr{C}^{\infty}(\Omega)$ such that $d_{x}=\lambda\left(a_{1}-a_{0}\right)$ and $d_{y}=\lambda\left(b_{1}-b_{0}\right)$.

(iii) The bundle curvatures $\tau_{0}, \tau_{1} \in \mathscr{C}^{\infty}(\Omega)$ of the two submersions coincide. 
Proof. It is easy to check that $f_{d}$ is an isometry if and only if $d$ satisfies (ii), so the equivalence between (i) and (ii) is proved. Since $f_{d}$ preserves the orientation in $\Omega \times \mathbb{R}$, we get that (i) implies (iii) from Remark 2.2. Finally, to prove that (iii) implies (ii), observe that $\tau_{0}=\tau_{1}$ means $\left(\lambda b_{0}\right)_{x}-\left(\lambda a_{0}\right)_{y}=\left(\lambda b_{1}\right)_{x}-\left(\lambda a_{1}\right)_{y}$ in view of (2-4). Equivalently, we have $\left(\lambda\left(a_{1}-a_{0}\right)\right)_{y}=\left(\lambda\left(b_{1}-b_{0}\right)\right)_{x}$, so (ii) follows from Poincaré's lemma and the fact that $\Omega$ is simply connected.

We remark that condition (i) in the statement is equivalent to the fact that the canonical examples for $\left(\lambda, a_{0}, b_{0}\right)$ and $\left(\lambda, a_{1}, b_{1}\right)$ represent the same Killing submersion for different initial sections. The function $d$ is, up to an additive constant, the vertical distance between such sections.

Lemma 2.7 is actually a local classification result for Killing submersions, since we have proved that all Killing submersions are locally equivalent to canonical examples. We will now give the general version.

Theorem 2.8 (uniqueness). For $i \in\{0,1\}$, let $\pi_{i}: \mathbb{E}_{i} \rightarrow M_{i}$ be a Killing submersion, $M_{i}$ being simply connected, with bundle curvature $\tau_{i} \in \mathscr{C}^{\infty}\left(M_{i}\right)$ for a given orientation in $\mathbb{E}_{i}$. Suppose that the fibers of $\pi_{0}$ and the fibers of $\pi_{1}$ have the same length and there exists an isometry $h: M_{0} \rightarrow M_{1}$. Let $p_{0} \in \mathbb{E}_{0}$ and $p_{1} \in \mathbb{E}_{1}$ be such that $h\left(\pi_{0}\left(p_{0}\right)\right)=\pi_{1}\left(p_{1}\right)$.

(a) If $\tau_{1} \circ h=\tau_{0}$, then there exists a unique orientation-preserving isometry $f: \mathbb{E}_{0} \rightarrow \mathbb{E}_{1}$ such that $\pi_{1} \circ f=h \circ \pi_{0}$ and $f\left(p_{0}\right)=p_{1}$.

(b) If $\tau_{1} \circ h=-\tau_{0}$, then there exists a unique orientation-reversing isometry $f: \mathbb{E}_{0} \rightarrow \mathbb{E}_{1}$ such that $\pi_{1} \circ f=h \circ \pi_{0}$ and $f\left(p_{0}\right)=p_{1}$.

Proof. Let us first consider the case of $M_{i}$ being a topological disk, so there exist conformal diffeomorphisms $\varphi_{i}: M_{i} \rightarrow \Omega$ such that $h \circ \varphi_{0}=\varphi_{1}$, where $\Omega \subset \mathbb{R}^{2}$ is an open set. For $i \in\{0,1\}$, Proposition 2.4 guarantees the existence of a global smooth section $F_{i}: M_{i} \rightarrow \mathbb{E}_{i}$ and a local diffeomorphism $f_{i}: \Omega \times \mathbb{R} \rightarrow \mathbb{E}_{i}$, given by $f_{i}(x, y, t)=\phi_{t}^{i}\left(F_{i}\left(\varphi_{i}^{-1}(x, y)\right)\right)$ as in Proposition 2.5, where $\left\{\phi_{t}^{i}\right\}$ is the 1parameter group of vertical translations associated to $\pi_{i}$. In other words, we obtain a commutative diagram as in Figure 1 , where $\pi: \Omega \times \mathbb{R} \rightarrow \Omega$ is the projection over the first factor.

Observe that $\varphi_{0}$ and $\varphi_{1}$ induce the same metric $\lambda^{2}\left(\mathrm{~d} x^{2}+\mathrm{d} y^{2}\right)$ on $\Omega$, and $f_{0}$ and $f_{1}$ induce canonical metrics $\mathrm{d} s_{\lambda, a_{0}, b_{0}}^{2}$ and $\mathrm{d} s_{\lambda, a_{1}, b_{1}}^{2}$ on $\Omega \times \mathbb{R}$, respectively. Moreover, the condition $\tau_{1} \circ h=\tau_{0}$ ensures that both canonical examples for $\left(\lambda, b_{0}, a_{0}\right)$ and $\left(\lambda, b_{1}, a_{1}\right)$ have the same bundle curvature, so Lemma 2.7 yields the existence of a isometry

$$
\hat{f}:\left(\Omega \times \mathbb{R}, \mathrm{d} s_{\lambda, a_{0}, b_{0}}^{2}\right) \rightarrow\left(\Omega \times \mathbb{R}, \mathrm{d} s_{\lambda, a_{1}, b_{1}}^{2}\right)
$$




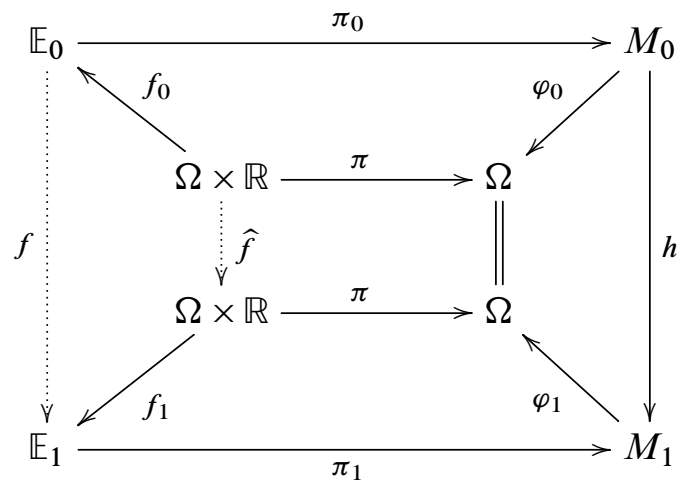

Figure 1. Horizontal and vertical arrows represent Killing submersions and isometries, respectively. Diagonal ones relate the original diagram with the canonical examples.

of the form $\hat{f}(x, y, z)=f(x, y, z+d(x, y))$ for some $d \in \mathscr{C}^{\infty}(\Omega)$, so $\pi \circ \hat{f}=\pi$. If $\tau_{1} \circ h=-\tau_{0}$, the canonical examples for $\left(\lambda, b_{0}, a_{0}\right)$ and $\left(\lambda, b_{1}, a_{1}\right)$ have opposite bundle curvatures, so it is easy to see that there exists an isometry of the form $\hat{f}(x, y, z)=f(x, y,-z-d(x, y))$ for some $d \in \mathscr{C}^{\infty}(\Omega)$.

In both cases, the isometry $\hat{f}$ induces an isometry from the quotient of

$$
\left(\Omega \times \mathbb{R}, \mathrm{d} s_{\lambda, a_{0}, b_{0}}^{2}\right)
$$

by a vertical translation to the quotient of $\left(\Omega \times \mathbb{R}, \mathrm{d} s_{\lambda, a_{1}, b_{1}}^{2}\right)$ by the same vertical translation. Adjusting the translation so that the length of the fibers of the quotient is the same as in $\mathbb{E}_{0}$ or $\mathbb{E}_{1}$, the isometry in the quotient provides an isometry $f: \mathbb{E}_{0} \rightarrow \mathbb{E}_{1}$ such that $\pi_{1} \circ f=h \circ \pi_{0}$. We get $f\left(p_{0}\right)=p_{1}$ by just composing $f$ with a vertical translation.

Finally, suppose that $M_{0}$ and $M_{1}$ are topological 2-spheres. Let $U_{0}=M_{0} \backslash\left\{q_{0}\right\}$ for some $q_{0} \neq \pi_{0}\left(p_{0}\right)$ and $U_{1}=h\left(U_{0}\right)=M_{1} \backslash\left\{h\left(q_{0}\right)\right\}$. Note that $h: U_{0} \rightarrow U_{1}$ is an isometry in the conditions of the disk case so it lifts to an isometry $f: V_{0} \rightarrow V_{1}$, where $V_{i}=\pi_{i}^{-1}\left(U_{i}\right)$ for $i \in\{0,1\}$, satisfying $\pi_{1} \circ f=h \circ \pi_{0}$ in $V_{0}$ and $f\left(p_{0}\right)=p_{1}$. Now, let $\tilde{p}_{0} \in V_{0}$ be such that $\pi_{0}\left(\tilde{p}_{0}\right) \neq \pi_{0}\left(p_{0}\right)$, and $\tilde{p}_{1}=f\left(\tilde{p}_{0}\right)$. Now take $\widetilde{q}_{0} \in M_{0}$ such that $\widetilde{q}_{0} \notin\left\{\pi_{0}\left(\tilde{p}_{0}\right), q_{0}\right\}$, and $\widetilde{U}_{0}=M_{0} \backslash\left\{\widetilde{q}_{0}\right\}, \widetilde{U}_{1}=h\left(\widetilde{U}_{0}\right)=M_{1} \backslash\left\{h\left(\widetilde{q}_{0}\right)\right\}$. The same reasoning above gives an isometry $\tilde{f}: \widetilde{V}_{0} \rightarrow \widetilde{V}_{1}$, where $\widetilde{V}_{i}=\pi_{i}^{-1}\left(\tilde{U}_{i}\right)$ for $i \in\{1,2\}$, satisfying the condition $\pi_{1} \circ \tilde{f}=h \circ \pi_{0}$ in $\tilde{V}_{0}$ and $\tilde{f}\left(\tilde{p}_{0}\right)=\tilde{p}_{1}=f\left(\tilde{p}_{0}\right)$.

Since $V=V_{0} \cap \widetilde{V}_{0}$ is connected, we have $f\left(\widetilde{p}_{0}\right)=\tilde{f}\left(\tilde{p}_{0}\right)$, and $(\mathrm{d} f)_{\tilde{p}_{0}}=(\mathrm{d} \widetilde{f})_{\tilde{p}_{0}}$ (because both $f$ and $\tilde{f}$ preserve the vertical direction and $\pi_{1} \circ \tilde{f}=h \circ \pi_{0}=\pi_{1} \circ f$ in $V$ ), and we conclude that $f=\tilde{f}$ in $V$. As $V_{0} \cup \tilde{V}_{0}=\mathbb{E}_{0}$, we deduce that $f$ can be extended (by $\tilde{f}$ ) to an isometry from $\mathbb{E}_{0}$ to $\mathbb{E}_{1}$, and it trivially satisfies the conditions in the statement. 
Killing isometries. We will now specialize some of the results in the previous section to study isometries of the total space of a Killing submersion $\pi: \mathbb{E} \rightarrow M$ preserving the Killing submersion structure, i.e., those preserving the direction of a unit vertical Killing vector field $\xi$.

Definition 2.9. In the previous notation, the isometries of $\mathbb{E}$ satisfying $f_{*} \xi=\xi$ or $f_{*} \xi=-\xi$ will be called Killing isometries.

The definition does not depend on the choice of $\xi$. If $f_{*} \xi=\xi$ (resp. $f_{*} \xi=-\xi$ ), then $f$ is said to preserve (resp. reverse) the orientation of the fibers. Note that preserving the orientation of the fibers is not related to preserving or reversing the orientation of the total space $\mathbb{E}$.

Lemma 2.10. Let $\pi: \mathbb{E} \rightarrow M$ be a Killing submersion with bundle curvature $\tau \in \mathscr{C}^{\infty}(M)$, and let $f: \mathbb{E} \rightarrow \mathbb{E}$ be a Killing isometry. Then:

(a) There exists a unique isometry $h: M \rightarrow M$ such that $\pi \circ f=h \circ \pi$.

(b) If $f$ preserves the orientation in $\mathbb{E}$, then $\tau \circ h=\tau$.

(c) If $f$ reverses the orientation in $\mathbb{E}$, then $\tau \circ h=-\tau$.

Proof. Item (a) follows from the fact that $f$ maps fibers to fibers and from the fact that $\mathrm{d} \pi$ is an isometry when restricted to the horizontal distribution. Now, it is easy to see that $(f, h)$ is an isomorphism of Killing submersions (see Definition 1.2), so (b) and (c) follow from Remark 2.2.

In fact, the map $f \mapsto h$ defined by (a) of Lemma 2.10 can be easily proved to be a group morphism from the group of Killing isometries to the group of isometries of $M$ with $\tau \circ h= \pm \tau$. Moreover, the normal subgroup of orientation-preserving isometries is mapped to those isometries of $M$ which preserve $\tau$. As an application of Theorem 2.8, we can prove that this morphism is surjective and its kernel consists of the vertical translations and, for $\tau \equiv 0$, also the symmetries with respect to a slice.

Corollary 2.11. Let $\pi: \mathbb{E} \rightarrow M$ be a Killing submersion with bundle curvature $\tau \in \mathscr{C}^{\infty}(M)$ and suppose that $M$ is simply connected. Let $h: M \rightarrow M$ be an isometry and take $p_{0}, q_{0} \in \mathbb{E}$ such that $h\left(\pi\left(p_{0}\right)\right)=\pi\left(q_{0}\right)$.

(a) If $\tau \circ h=\tau$ in $M$, then there exists a unique orientation-preserving Killing isometry $f: \mathbb{E} \rightarrow \mathbb{E}$ such that $\pi \circ f=h \circ \pi$ and $f\left(p_{0}\right)=q_{0}$.

(b) If $\tau \circ h=-\tau$ in $M$, then there exists a unique orientation-reversing Killing isometry $f: \mathbb{E} \rightarrow \mathbb{E}$ such that $\pi \circ f=h \circ \pi$ and $f\left(p_{0}\right)=q_{0}$.

As an immediate consequence, in the following two situations there do not exist Killing isometries reversing the orientation of the total space:

- If the bundle curvature is a nonzero constant.

- If $M$ is a Riemannian 2-sphere and $\int_{M} \tau \neq 0$. 


\section{Curves in Killing submersions}

\section{The horizontal lift of a curve.}

Definition 3.1. Let $\pi: \mathbb{E} \rightarrow M$ be a Killing submersion and $\alpha:[c, d] \rightarrow M$ a $\mathscr{C}^{1}$-curve. A horizontal (or Legendrian) lift of $\alpha$ is a $\mathscr{C}^{1}$-curve $\widetilde{\alpha}:[c, d] \rightarrow \mathbb{E}$ such that $\tilde{\alpha}^{\prime}$ is always horizontal and $\pi \circ \tilde{\alpha}=\alpha$ in $[c, d]$.

This concept extends to piecewise $\mathscr{C}^{1}$-curves $\alpha:[c, d] \rightarrow M$, i.e., $\alpha$ such that there is a partition $c=t_{0}<t_{1}<\ldots<t_{n}=d$ so that $\alpha_{\mid\left[t_{i-1}, t_{i}\right]}$ is $\mathscr{C}^{1}$ for all $i \in\{1, \ldots, n\}$. A horizontal lift of $\alpha$ is a continuous curve $\tilde{\alpha}:[c, d] \rightarrow \mathbb{E}$ such that $\tilde{\alpha}_{\mid\left[t_{i-1}, t_{i}\right]}$ is a horizontal lift of $\alpha_{\mid\left[t_{i-1}, t_{i}\right]}$ for all $i \in\{1, \ldots, n\}$.

Lemma 3.2. Let $\alpha:[c, d] \rightarrow M$ be a piecewise $\mathscr{C}^{1}$-curve. Given $p_{0} \in \mathbb{E}$ such that $\pi\left(p_{0}\right)=\alpha(c)$, there exists a unique horizontal lift $\tilde{\alpha}$ of $\alpha$ such that $\widetilde{\alpha}(c)=p_{0}$.

Proof. Let $c=t_{0}<t_{1}<\ldots<t_{n}=d$ be a partition such that $\alpha_{\mid\left[t_{i-1}, t_{i}\right]}$ is a $\mathscr{C}^{1}$-curve. We can refine the partition so that $\alpha\left(\left[t_{i-1}, t_{i}\right]\right) \subset U_{i}$ for some conformal chart $\left(U_{i}, \varphi_{i}\right)$ of $M$ for all $i$. Thus, we can assume that $\alpha$ is contained in such a chart $(U, \varphi)$, so $\widetilde{\alpha}$ will be contained in $\pi^{-1}(U)$.

This allows us to work in the canonical example given in Definition 2.6 for $\Omega=\varphi(U)$ and some $\lambda, a, b \in \mathscr{C}^{\infty}(\varphi(U))$ with $\lambda>0$. Writing in coordinates $\alpha(t)=(x(t), y(t)) \in \varphi(U)$, a horizontal lift of $\alpha$ must be of the form $\widetilde{\alpha}(t)=$ $(x(t), y(t), z(t))$ for some $z:[c, d] \rightarrow \mathbb{R}$, and must satisfy $\left\langle\widetilde{\alpha}^{\prime}, \partial_{z}\right\rangle=0$. This last condition can be developed as

$$
z^{\prime}=\lambda(x, y) \cdot\left(a(x, y) x^{\prime}+b(x, y) y^{\prime}\right) .
$$

Since $\pi\left(p_{0}\right)=\alpha(c)$, we have $p_{0}=\left(x(c), y(c), z_{0}\right)$ for some $z_{0} \in \mathbb{R}$. We deduce that there exists a unique $\mathscr{C}^{1}$-function $z(t)$ satisfying (3-1) with initial condition $z(c)=z_{0}$, so the horizontal lift exists and is unique.

We can now give a geometric meaning of the bundle curvature in terms of the difference of heights of the endpoints of the horizontal lift of closed curves (see also [Daniel et al. 2009, Proposition 1.6.2]). Supposing that the fibers have infinite length will be necessary for the difference of heights to make sense.

Proposition 3.3. Let $\pi: \mathbb{E} \rightarrow M$ be a Killing submersion whose fibers have infinite length. Given a simple piecewise $\mathscr{b}^{1}$-curve $\alpha:[c, d] \rightarrow M$ bounding an orientable relatively compact open set $G \subset M$ and a horizontal lift $\tilde{\alpha}$ of $\alpha$, we have

$$
\left|\int_{G} \tau\right|=\frac{h}{2}
$$

where $h$ is the length of the vertical segment joining $\widetilde{\alpha}(c)$ and $\widetilde{\alpha}(d)$. 
Proof. Let us consider an atlas of $M$ consisting of conformal charts. We will first suppose that $\bar{G}$ is contained in one of the charts $(U, \varphi)$, so we can suppose that we are working in the canonical example given by Definition 2.6 for $\lambda, a, b \in \mathscr{C}^{\infty}(\varphi(U))$ with $\lambda>0$. Moreover, (2-4) allows us to write $\tau$ as a divergence in $\varphi(G)$. The divergence theorem yields

$$
\int_{\varphi(G)} 2 \tau=\int_{\varphi(G)} \operatorname{div}\left(\frac{b}{\lambda} \partial_{x}-\frac{a}{\lambda} \partial_{y}\right)=\int_{\partial \varphi(G)}\left\langle\frac{b}{\lambda} \partial_{x}-\frac{a}{\lambda} \partial_{y}, \eta\right\rangle,
$$

where $\eta$ is the outer unit conormal to $\varphi(G)$ along its boundary. We write in coordinates $\alpha=(x, y)$ and $\tilde{\alpha}=(x, y, z)$, and suppose $\alpha$ is parametrized by arclength (i.e., $\left.\left(x^{\prime}\right)^{2}+\left(y^{\prime}\right)^{2}=1 / \lambda^{2}\right)$. Hence $\eta=-y^{\prime} \partial_{x}+x^{\prime} \partial_{y}$, up to a sign, so we deduce from (3-1) that

$$
\left|\int_{G} 2 \tau\right|=\left|\int_{c}^{d} \lambda \cdot\left(a x^{\prime}+b y^{\prime}\right)\right|=\left|\int_{c}^{d} z^{\prime}\right|=|z(d)-z(c)| .
$$

As $h=|z(d)-z(c)|$ in this model, we are done.

If $\bar{G}$ does not lie in a single chart, we can triangulate $\bar{G}$ by a finite number of triangles with piecewise $\mathscr{C}^{1}$ boundaries so each triangle is contained in a coordinate chart of the atlas (see, for instance, the proof of [Jost 2002, Theorem 2.3.A.1]) and $\alpha$ can be expressed as a finite sum of the boundaries of these triangles. As $G$ is orientable, such boundaries can be oriented so that the interior ones cancel out in pairs. The argument above applied to each triangle together with the divergence theorem gives the desired result.

Geodesics. Let $\pi: \mathbb{E} \rightarrow M$ be a Killing submersion. Given two vector fields $X, Y \in \mathfrak{X}(M)$, we can consider their horizontal lifts $\bar{X}, \bar{Y} \in \mathfrak{X}(\mathbb{E})$. Then the following equality holds (see [do Carmo 1992, pp. 185-187]):

$$
\bar{\nabla} \bar{X} \bar{Y}=\overline{\nabla_{X} Y}+[\bar{X}, \bar{Y}]^{v},
$$

where $\nabla$ and $\bar{\nabla}$ are the Levi-Civita connections in $M$ and $\mathbb{E}$, respectively, $\overline{\nabla_{X} Y}$ is the horizontal lift of $\nabla_{X} Y$ and $[\bar{X}, \bar{Y}]^{v}$ is the vertical part of $[\bar{X}, \bar{Y}]$.

From (3-2) we deduce that the horizontal lift of a geodesic in $M$ is a geodesic in $\mathbb{E}$. Since not all geodesics are horizontal or vertical, we will need a slight improvement of this argument to classify them all.

Lemma 3.4. Geodesics in $\mathbb{E}$ make a constant angle with a vertical Killing vector field $\xi$.

Proof. Given a geodesic $\gamma$ in $\mathbb{E}$, we can compute

$$
\frac{d}{d t}\left\langle\gamma^{\prime}, \xi\right\rangle=\left\langle\bar{\nabla}_{\gamma^{\prime}} \gamma^{\prime}, \xi\right\rangle+\left\langle\gamma^{\prime}, \bar{\nabla}_{\gamma^{\prime}} \xi\right\rangle
$$


The first term on the right-hand side vanishes since $\gamma$ is a geodesic, and the second one also vanishes because $\bar{\nabla}_{\gamma^{\prime}} \xi=\tau \gamma^{\prime} \wedge \xi$ (see Lemma 2.1).

Given a real number $\mu \in \mathbb{R}$ and a smooth curve $\alpha:[a, b] \rightarrow M$, we can consider the smooth curve

$$
\gamma:[a, b] \rightarrow \mathbb{E}, \quad \gamma(t)=\phi_{\mu t}(\tilde{\alpha}(t)),
$$

where $\left\{\phi_{t}\right\}$ is the group of vertical translations associated to a unit vertical vector field $\xi$. The chain rule allows us to compute

$$
\gamma^{\prime}(t)=\mu \xi_{\gamma(t)}+\left(d \phi_{\mu t}\right) \tilde{\alpha}(t)\left(\widetilde{\alpha}^{\prime}(t)\right)
$$

so $\gamma$ makes a constant angle with $\xi$ and will be our candidate to geodesic. Taking into account that $\left[\widetilde{\alpha}^{\prime}, \xi\right]=0$ and (3-2), we get

$$
\bar{\nabla}_{\gamma^{\prime}} \gamma^{\prime}=2 \mu \tau \widetilde{\alpha}^{\prime} \wedge \xi+\overline{\nabla_{\alpha^{\prime}} \alpha^{\prime}}
$$

Let us suppose that $\alpha$ has unit speed and consider $J$ the $\pm(\pi / 2)$-rotation in $T M$ (the sign will be chosen below). Then there exists a function $\kappa_{g}:[a, b] \rightarrow \mathbb{R}$, the geodesic curvature, such that $\nabla_{\alpha^{\prime}} \alpha^{\prime}=\kappa_{g} \cdot J \alpha^{\prime}$. The horizontal lift of $J \alpha^{\prime}$ is a horizontal and unitary vector field along $\tilde{\alpha}$, orthogonal to $\tilde{\alpha}^{\prime}$. Hence, we can choose the sign of $J$ so the horizontal lift of $J \alpha^{\prime}$ is equal to $-\widetilde{\alpha}^{\prime} \wedge \xi$. Now (3-4) implies that $\gamma$ is a geodesic if and only if

$$
\kappa_{g}(t)=2 \mu \tau(\alpha(t)) .
$$

Lemma 3.5. Given $\mu \in \mathbb{R}, p \in M$ and $v \in T_{p} M$, there exist $\varepsilon>0$ and a unique unit-speed smooth curve $\alpha:]-\varepsilon, \varepsilon\left[\rightarrow M\right.$ such that $\alpha(0)=p, \alpha^{\prime}(0)=v$ and satisfying (3-5).

Moreover, if $M$ is complete then $\alpha$ extends to the whole real line.

Proof. We will work in a conformal parametrization $\varphi: U \subset \mathbb{R}^{2} \rightarrow M$ compatible with the orientation fixed above, where $U$ is a neighborhood of $p$. Then we identify $\alpha$ with the coordinates $(x, y)=\varphi^{-1} \circ \alpha$. Since $\alpha$ has unit speed, there must exist a smooth function $\theta$ such that $x^{\prime}=\lambda^{-1} \cos \theta$ and $y^{\prime}=\lambda^{-1} \sin \theta$, where $\lambda$ denotes the conformal factor. The geodesic curvature of $\alpha$ with respect to $J \alpha^{\prime}=-y^{\prime} \partial_{x}+x^{\prime} \partial_{y}$ is given by

$$
\kappa_{g}=\theta^{\prime}+\frac{\lambda_{x}}{\lambda^{2}} \sin \theta-\frac{\lambda y}{\lambda^{2}} \cos \theta .
$$

Now, (3-5) becomes the first-order ODE system 


$$
\left\{\begin{aligned}
x^{\prime} & =\frac{1}{\lambda(x, y)} \cos \theta, \\
y^{\prime} & =\frac{1}{\lambda(x, y)} \sin \theta, \\
\theta^{\prime} & =2 \mu \tau(x, y)-\frac{\lambda_{x}(x, y)}{\lambda(x, y)^{2}} \sin \theta+\frac{\lambda_{y}(x, y)}{\lambda^{2}(x, y)} \cos \theta .
\end{aligned}\right.
$$

The general theory of ODEs guarantees the existence of a unique smooth solution in a neighborhood of the origin when prescribing $\alpha(0), \alpha^{\prime}(0)$ (note that these initial data are equivalent to $x(0), y(0)$ and $\theta(0))$. Observe that the solution can be extended as long as $\alpha$ is contained in $U$, so if $M$ is complete and we take an atlas consisting of conformal parametrizations compatible with the orientation, then $\alpha$ extends to the whole real line.

It is important to notice that the curve $\gamma$ given by Lemma 3.5 satisfies $\left\|\gamma^{\prime}\right\|^{2}=$ $1+\mu^{2}$, so after a reparametrization by arc-length, we obtain $\left\langle\gamma^{\prime}, \xi\right\rangle=\mu / \sqrt{1+\mu^{2}}$. This last expression varies in ] $-1,1[$ when $\mu \in \mathbb{R}$, so this construction covers all geodesics in $\mathbb{E}$, except for the vertical ones.

Proposition 3.6. Given $p \in \mathbb{E}$, all geodesics in $\mathbb{E}$ passing through $p$ are of one (and only one) of the following types:

(1) vertical geodesics (fibers of the submersion),

(2) horizontal lifts of geodesics in $M$ passing through $\pi(p)$,

(3) of the form $\gamma(t)=\phi_{\mu t}(\tilde{\alpha}(t))$, where $\tilde{\alpha}$ is a horizontal lift of $\alpha$ in $M$ such that $\alpha(0)=\pi(p)$ and satisfying (3-5) for some $\mu \neq 0$.

In particular, if $M$ is complete, then so is $\mathbb{E}$.

Remark 3.7. When the bundle curvature is constant, nonvertical geodesics project into curves with constant geodesic curvature. Moreover, the geodesic is horizontal if and only if its projection is also a geodesic. This gives an easy way to compute geodesics in the $\mathbb{E}(\kappa, \tau)$-spaces.

\section{Existence results}

When the base is simply connected, Theorem 2.8 gives a uniqueness result for Killing submersions; in this section we will investigate the existence problem and prove that we can fix beforehand any bundle curvature under the same assumption of simple connectedness.

Killing submersions over a disk. Given an open set $\Omega \subset \mathbb{R}^{2}$ and $\lambda, \tau \in \mathscr{C}^{\infty}(\Omega)$ with $\lambda>0$, we wonder whether it is possible to solve for $a$ and $b$ in (2-4). An explicit way of doing so when $\Omega$ is star-shaped is given in the following lemma by just taking $\delta=2 \lambda^{2} \tau$. 
Lemma 4.1. Let $\Omega \subset \mathbb{R}$ be open and star-shaped with respect to the origin, and $\delta \in \mathscr{C}^{\infty}(\Omega)$. Then $\eta \in \mathscr{C}^{\infty}(\Omega)$, given by

$$
\eta(x, y)=\int_{0}^{1} s \delta(x s, y s) \mathrm{d} s,
$$

satisfies the identity $\delta=(x \eta)_{x}+(y \eta)_{y}$.

Proof. It is a direct computation.

Theorem 4.2. Let $\Omega \subseteq \mathbb{R}^{2}$ be an open set star-shaped with respect to the origin and $\lambda, \tau \in \mathscr{C}^{\infty}(\Omega)$ with $\lambda>0$. If $\pi: \mathbb{E} \rightarrow\left(\Omega, \lambda^{2}\left(\mathrm{~d} x^{2}+\mathrm{d} y^{2}\right)\right)$ is a Killing submersion with bundle curvature $\tau$ and $\mathbb{E}$ is simply connected, then it is isomorphic to the canonical example

$$
\begin{gathered}
\pi_{1}:\left(\Omega \times \mathbb{R}, \mathrm{d} s^{2}\right) \rightarrow\left(\Omega, \lambda^{2}\left(\mathrm{~d} x^{2}+\mathrm{d} y^{2}\right)\right), \quad \pi_{1}(x, y, z)=(x, y), \\
\mathrm{d} s^{2}=\lambda(x, y)^{2}\left(\mathrm{~d} x^{2}+\mathrm{d} y^{2}\right)+(\mathrm{d} z+\eta(x, y)(y \mathrm{~d} x-x \mathrm{~d} y))^{2},
\end{gathered}
$$

where the function $\eta \in \mathscr{C}^{\infty}(\Omega)$ is given by

$$
\eta(x, y)=2 \int_{0}^{1} s \tau(x s, y s) \lambda(x s, y s)^{2} \mathrm{~d} s .
$$

Remark 4.3. Note that star-shapeness makes everything explicit but an existence and uniqueness theorem also holds in the (more general) simply connected case. It suffices to conformally parametrize such a simply connected domain by a disk and apply Theorem 4.2.

Remark 4.4. If we drop the condition that $\mathbb{E}$ is simply connected, it can be easily shown that any Killing submersion $\pi: \mathbb{E} \rightarrow \Omega$ is isomorphic to a Riemannian quotient of the Killing submersion constructed in Theorem 4.2 by a vertical translation. In particular, $\mathbb{E}$ is diffeomorphic to $\Omega \times \mathbb{S}^{1}$.

It is interesting to specialize Theorem 4.2 to the case $M=\mathbb{M}^{2}(\kappa)$, the complete simply connected surface with constant Gaussian curvature $\kappa \in \mathbb{R}$, to get models for all Killing submersions over $\mathbb{R}^{2}, \mathbb{H}^{2}(\kappa)$ and $\mathbb{S}^{2}(\kappa)$ minus a point. Given $\kappa \in \mathbb{R}$, we define $\lambda_{\kappa} \in \mathscr{C}^{\infty}\left(\Omega_{\kappa}\right)$ as

where

$$
\lambda_{\kappa}(x, y)=\left(1+\frac{\kappa}{4}\left(x^{2}+y^{2}\right)\right)^{-1},
$$

$$
\Omega_{k}= \begin{cases}\left\{(x, y) \in \mathbb{R}^{2}: x^{2}+y^{2}<-4 / \kappa\right\} & \text { if } \kappa<0, \\ \mathbb{R}^{2} & \text { if } \kappa \geq 0 .\end{cases}
$$

Then the metric $\lambda_{\kappa}^{2}\left(\mathrm{~d} x^{2}+\mathrm{d} y^{2}\right)$ in $\Omega_{\kappa}$ has constant Gaussian curvature $\kappa$. If $\tau$ is constant, then $\eta=\tau \lambda_{\kappa}$ in (4-1), and we obtain the metrics of the spaces 
$\mathbb{E}(\kappa, \tau) \equiv \Omega_{\kappa} \times \mathbb{R}$ given in [Daniel 2007, Section 2.3]:

$$
\lambda_{\kappa}^{2}\left(\mathrm{~d} x^{2}+\mathrm{d} y^{2}\right)+\left(\mathrm{d} z+\tau \lambda_{\kappa}(y \mathrm{~d} x-x \mathrm{~d} y)\right)^{2} .
$$

Recall that we are not considering a whole fiber of a point in $\mathbb{S}^{2}(\kappa)$ for $\kappa>0$. The global case will be treated in the next section.

Killing submersions over a 2-sphere. We can define Killing submersions over $\mathbb{S}^{2}$ as different as the Riemannian products $\mathbb{S}^{2} \times \mathbb{R}$ and $\mathbb{S}^{2} \times \mathbb{S}^{1}$ (both with $\tau=0$ ) or the Berger spheres and the lens spaces $L(n, 1)$ via the Hopf projection (see Remark 4.8 below). Throughout this section, we will suppose that the surface playing the role of base surface is $\left(\mathbb{S}^{2}, g\right)$ for some Riemannian metric $g$.

Unlike in the cases treated above, this surface is compact. Hence, given a Killing submersion $\pi: \mathbb{E} \rightarrow\left(\mathbb{S}^{2}, g\right)$ and its bundle curvature $\tau \in \mathscr{C}^{\infty}(M)$, the total bundle curvature

$$
T=\int_{M} \tau
$$

is well-defined and finite. This quantity will make the difference between the possible topologies of the total space.

The case $T=0$.

Proposition 4.5. Let $\pi: \mathbb{E} \rightarrow\left(\mathbb{S}^{2}, g\right)$ be a Killing submersion with total bundle curvature $T=0$. Then the submersion admits a global smooth section.

(a) If the length of the fibers of $\pi$ is infinite, then it is isomorphic to

$$
\pi_{1}:\left(\mathbb{S}^{2} \times \mathbb{R}, \mathrm{d} s^{2}\right) \rightarrow\left(\mathbb{S}^{2}, g\right), \quad \pi_{1}(p, t)=p,
$$

for some Riemannian metric $\mathrm{d} s^{2}$ defined in $\mathbb{S}^{2} \times \mathbb{R}$ and such that $\partial_{t}$ is a unit vertical Killing vector field.

(b) Otherwise, the Killing submersion is isomorphic to the Riemannian quotient of the example in (a) by some vertical translation.

Proof. The condition $T=0$ guarantees the existence of an equator $\Gamma \subset \mathbb{S}^{2}$ such that $D_{1}$ and $D_{2}$, the two open components of $\mathbb{S}^{2} \backslash \Gamma$, satisfy

$$
\int_{D_{1}} \tau=\int_{D_{2}} \tau=0
$$

Let $\widetilde{\Gamma} \subset \mathbb{E}$ be any horizontal lift of $\Gamma$. If the fibers of $\pi$ have infinite length, then Proposition 3.3 implies that $\widetilde{\Gamma}$ is a closed curve in $\mathbb{E}$. For $i \in\{1,2\}$, as $\widetilde{\Gamma}$ lies in the boundary of $\pi^{-1}\left(\bar{D}_{i}\right)$ and projects one-to-one by $\pi$ onto $\Gamma$, there exists a section $F_{i}: \bar{D}_{i} \rightarrow \mathbb{E}$ with $F_{i}(\Gamma)=\widetilde{\Gamma}$. Thus $F: \mathbb{S}^{2} \rightarrow \mathbb{E}$ defined by $F=F_{i}$ in $\bar{D}_{i}$ is a global continuous section, and there is no loss of generality in supposing that $F$ is smooth (just by perturbing it in a neighborhood of $\Gamma$ ). Then $\Psi: \mathbb{S}^{2} \times \mathbb{R} \rightarrow \mathbb{E}$ 
given by $\Psi(p, t)=\phi_{t}(F(p))$ is a global diffeomorphism, where $\phi_{t}$ denotes the 1-parameter group of vertical isometries. The induced metric $\mathrm{d} s^{2}$ in $\mathbb{S}^{2} \times \mathbb{R}$ through $\Psi$ satisfies the requirements of (a).

In the case that the length of the fibers of $\pi$ is finite, we can work in the universal cover of $\pi^{-1}\left(\bar{D}_{i}\right)$, for $i \in\{1,2\}$, repeat the arguments above, and finally take a convenient quotient by a vertical translation.

The rest of this section is devoted to obtaining explicit models for the metrics in $\mathbb{S}^{2} \times \mathbb{R}$ making the projection over the first factor a Killing submersion. This will show that Proposition 4.5 is sharp, but we will also obtain a quite general method for constructing Killing submersions.

Inspired by the canonical metrics in (2-2), let us consider arbitrary functions $a_{1}, \ldots, a_{n} \in \mathscr{C}^{\infty}\left(\mathbb{R}^{n}\right)$ and the projection over the first $n$ coordinates

$$
\pi:\left(\mathbb{R}^{n+1}, \mathrm{~d} s^{2}\right) \rightarrow \mathbb{R}^{n}
$$

Here, we endow $\mathbb{R}^{n}$ with the usual metric and

$$
\mathrm{d} s^{2}=\sum_{k=1}^{n} \mathrm{~d} x_{i}^{2}+\left(\mathrm{d} t-\sum_{k=1}^{n} a_{k} \mathrm{~d} x_{k}\right)^{2},
$$

where we denote by $\left(x_{1}, \ldots, x_{n}, t\right)$ the usual coordinates of $\mathbb{R}^{n+1}$. Then $\pi$ is a Riemannian submersion whose fibers are the integral curves of the unit Killing vector field $\partial_{t}$ in $\left(\mathbb{R}^{n+1}, \mathrm{~d} s^{2}\right)$.

Given a smooth orientable surface $\Sigma$, we can isometrically embed it in $\mathbb{R}^{n}$ for some $n \in \mathbb{N}$ by the Nash embedding theorem [1956]. Then we shall consider the metric induced by (4-2) in $\Sigma \times \mathbb{R} \subset \mathbb{R}^{n+1}$. Obviously, $\pi$ restricts to a Killing submersion $\Sigma \times \mathbb{R} \rightarrow \Sigma$. We will now compute its bundle curvature in terms of the functions $a_{k}$, but we will first need a convention for the orientation in $\Sigma \times \mathbb{R}$ : if a local frame $\left\{e_{1}, e_{2}\right\}$ in $\Sigma$ is positively oriented, then $\left\{E_{1}, E_{2}, \partial_{t}\right\}$ will be said positively oriented in $\Sigma \times \mathbb{R}$, where $E_{i}$ is the horizontal lift of $e_{i}$ for $i \in\{1,2\}$.

Proposition 4.6. Let $\Sigma$ be a smooth oriented surface isometrically embedded in $\mathbb{R}^{n}$. The Killing submersion $\Sigma \times \mathbb{R} \rightarrow \Sigma$ defined above has bundle curvature

$$
\tau=\frac{1}{2} \operatorname{div}_{\Sigma}(J T),
$$

where $T=\left(\partial_{t}\right)^{\top} \in \mathfrak{X}(\Sigma)$ is the component of $\partial_{t}$ tangent to $\Sigma \equiv \Sigma \times\{0\} \subset \mathbb{R}^{n+1}$ with respect to $\mathrm{d} s^{2}$, and $J: \mathfrak{X}(\Sigma) \rightarrow \mathfrak{X}(\Sigma)$ is a $(\pi / 2)$-rotation in $T \Sigma$.

Proof. Let $X: \Omega \subset \mathbb{R}^{2} \rightarrow \Sigma$ be a local conformal parametrization of $\Sigma$ with conformal factor $\lambda \in \mathscr{C}^{\infty}(\Omega)$, and such that $\left\{(1 / \lambda) X_{u},(1 / \lambda) X_{v}\right\}$ is a positively oriented orthonormal frame of $T \Sigma$. Let $\left\{E_{1}, E_{2}\right\} \subset \mathfrak{X}(X(\Omega) \times \mathbb{R})$ be a horizontal lift of the frame $\left\{(1 / \lambda) X_{u},(1 / \lambda) X_{v}\right\}$ which, together with $E_{3}=\partial_{t}$, is a positively 
oriented orthonormal frame in $X(\Omega) \times \mathbb{R}$. As in (2-4), we can compute $\tau$ from the identity $2 \tau=\left\langle\left[E_{1}, E_{2}\right], E_{3}\right\rangle$. Note that there exist $f, g \in \mathscr{C}^{\infty}(X(\Omega))$ such that $E_{1}=(1 / \lambda) X_{u}+f \partial_{t}$ and $E_{2}=(1 / \lambda) X_{v}+g \partial_{t}$, so

$$
\begin{aligned}
{\left[E_{1}, E_{2}\right] } & =\left[\frac{1}{\lambda} X_{u}, \frac{1}{\lambda} X_{v}\right]+\left[\frac{1}{\lambda} X_{u}, g \partial_{t}\right]+\left[f \partial_{t}, \frac{1}{\lambda} X_{u}\right]+\left[f \partial_{t}, g \partial_{t}\right] \\
& =\frac{1}{\lambda^{3}}\left(\lambda_{v} X_{u}-\lambda_{u} X_{v}\right)+\frac{1}{\lambda}\left(g_{u}-f_{v}\right) \partial_{t} .
\end{aligned}
$$

Moreover, since $0=\left\langle E_{1}, E_{3}\right\rangle=\left\langle(1 / \lambda) X_{u}+f \partial_{t}, \partial_{t}\right\rangle$, we deduce that $\left\langle X_{u}, \partial_{t}\right\rangle=$ $-\lambda f$ and, analogously, $\left\langle X_{v}, \partial_{t}\right\rangle=-\lambda g$. Hence,

$$
2 \tau=\left\langle\left[E_{1}, E_{2}\right], E_{3}\right\rangle=\frac{1}{\lambda^{2}}\left((\lambda g)_{u}-(\lambda f)_{v}\right)=\operatorname{div}_{\Sigma}(Y),
$$

where $Y \in \mathfrak{X}(\Sigma)$ is the vector field $(g / \lambda) X_{u}-(f / \lambda) X_{v}$. From here, it is easy to check that $Y=J T$ and we are done.

Remark 4.7. If $\Sigma$ is compact, then $\int_{\Sigma} \tau=(1 / 2) \int_{\Sigma} \operatorname{div}(J T)=0$ as an application of the divergence theorem. Conversely, every function on a compact orientable surface $\Sigma$ with zero integral is well-known to be the divergence of some vector field on $\Sigma$.

As a particular case, we may consider the round sphere

$$
\mathbb{S}^{2}(\kappa)=\left\{(x, y, z) \in \mathbb{R}^{3}: x^{2}+y^{2}+z^{2}=\frac{1}{\kappa}\right\} \subset \mathbb{R}^{3},
$$

and endow $\mathbb{S}^{2} \times \mathbb{R} \subset \mathbb{R}^{4}$ with the metric given by (4-2) for $n=3$ and some $a_{1}, a_{2}, a_{3} \in \mathscr{C}^{\infty}\left(\mathbb{R}^{3}\right)$. The stereographic projection $X: \mathbb{R}^{2} \rightarrow \mathbb{S}^{2}(\kappa) \backslash\{(0,0,1 / \sqrt{\kappa})\}$ defined by

$$
X(u, v)=\left(\frac{2 u}{\kappa\left(u^{2}+v^{2}\right)+1}, \frac{2 v}{\kappa\left(u^{2}+v^{2}\right)+1}, \frac{1}{\sqrt{\kappa}} \frac{\kappa\left(u^{2}+v^{2}\right)-1}{\kappa\left(u^{2}+v^{2}\right)+1}\right)
$$

allows us to work out the bundle curvature $\tau$ of the induced Killing submersion $\mathbb{S}^{2}(\kappa) \times \mathbb{R} \rightarrow \mathbb{S}^{2}(\kappa)$ as in the proof of Proposition 4.6. We get

$$
2 \tau=\sqrt{\kappa}\left(\left(y a_{3}-z a_{2}\right)_{x}+\left(z a_{1}-x a_{3}\right)_{y}+\left(x a_{2}-y a_{1}\right)_{z}\right) .
$$

The case $T \neq 0$. Let us consider the 3 -sphere

$$
\mathbb{S}^{3}=\left\{(z, w) \in \mathbb{C}^{2}:|z|^{2}+|w|^{2}=1\right\} \subset \mathbb{C}^{2},
$$

and $\mathbb{S}^{2}(\kappa)=\left\{(z, t):|z|^{2}+t^{2}=1 / \kappa\right\} \subset \mathbb{C} \times \mathbb{R}$ for $\kappa>0$. The submersion

$$
\pi_{\mathrm{Hopf}}: \mathbb{S}^{3} \rightarrow \mathbb{S}^{2}(\kappa), \quad(z, w) \mapsto \frac{1}{\sqrt{\kappa}}\left(2 z \bar{w},|z|^{2}-|w|^{2}\right),
$$

is known as the Hopf projection. The fiber passing through $(z, w) \in \mathbb{S}^{3}$ is given 
by $\left\{\left(e^{i t} z, e^{i t} w\right): t \in \mathbb{R}\right\}$ and the orbit of a point under the 1-parameter group of diffeomorphisms

$$
\phi_{t}(z, w)=\left(e^{i t} z, e^{i t} w\right), \quad t \in \mathbb{R},
$$

coincides with its fiber with respect to the submersion.

Remark 4.8. Given a natural number $n \in \mathbb{N}$, we can consider the quotient of $\mathbb{S}^{3}$ under the group of diffeomorphisms $G_{n}=\left\{\phi_{2 \pi k / n}: k \in\{1, \ldots, n\}\right\}$, which is cyclic and has order $n$. The quotient $\mathbb{S}^{3} / G_{n}$ is known as the lens space $L(n, 1)$. The condition $\pi_{\text {Hopf }} \circ \phi_{t}=\pi_{\text {Hopf }}$ guarantees that $\pi_{\text {Hopf }}$ induces a submersion $\pi_{n}: L(n, 1) \rightarrow \mathbb{S}^{2}(\kappa)$. Observe that, for any $n \in \mathbb{N}$, the space $L(n, 1)$ is orientable and its fundamental group is isomorphic to the cyclic group of order $n$, so two lens spaces $L(n, 1)$ and $L(m, 1)$ are not homeomorphic for $m \neq n$ (see [Saveliev 1999] for a more detailed description).

If we endow $\mathbb{S}^{3}$ with a metric making $\pi_{\mathrm{Hopf}}$ a Killing submersion, then the fibers of $\pi_{\text {Hopf }}$ have finite length (they are compact) and it is easy to check that $\pi_{n}$ is a Killing submersion when we consider the quotient metric, for all $n$. Moreover, the length of the fibers of $\pi_{\mathrm{Hopf}}$ in $\mathbb{S}^{3}$ is $n$ times the length of the corresponding fibers of $\pi_{n}$ in $L(n, 1)$.

Proposition 4.9. Let $\pi: \mathbb{E} \rightarrow\left(\mathbb{S}^{2}, g\right)$ be a Killing submersion with total bundle curvature $T \neq 0$. Then there exists $n \in \mathbb{N}$ such that the length of the fibers is equal to $|2 T| / n$.

(a) If $n=1$, then $\pi: \mathbb{E} \rightarrow\left(\mathbb{S}^{2}, g\right)$ is isomorphic to the Hopf fibration

$$
\pi_{\text {Hopf }}:\left(\mathbb{S}^{3}, \mathrm{~d} s^{2}\right) \rightarrow\left(\mathbb{S}^{2}, g\right), \quad \pi_{\text {Hopf }}(z, w)=\left(2 z \bar{w},|z|^{2}-|w|^{2}\right),
$$

for some Riemannian metric $\mathrm{d} s^{2}$ in $\mathbb{S}^{3}$ such that $\xi_{(z, w)}=(\pi / T)(i z, i w)$ is a unit Killing vector field.

(b) If $n>1$, then $\pi: \mathbb{E} \rightarrow\left(\mathbb{S}^{2}, g\right)$ is isomorphic to the Riemannian quotient of a submersion as in (a) by a vertical translation of length $|2 T| / n$.

Proof. As in the proof of Proposition 4.5, let us take a geodesic $\Gamma$ which divides $\mathbb{S}^{2}$ in two hemispheres $D_{1}$ and $D_{2}$ such that

$$
\int_{D_{1}} \tau=\int_{D_{2}} \tau=\frac{T}{2}
$$

We parametrize $\Gamma$ as $\gamma:[a, b] \rightarrow \mathbb{S}^{2}$ and a horizontal lift $\tilde{\Gamma}$ of $\Gamma$ as $\tilde{\gamma}:[a, b] \rightarrow \mathbb{E}$. The universal Riemannian covering space of $\pi^{-1}\left(\bar{D}_{i}\right)$, for $i \in\{1,2\}$, will be denoted by $W_{i} \equiv \bar{D}_{i} \times \mathbb{R}$, and is a closed solid cylinder. The curve $\widetilde{\Gamma}$ can be lifted to both $W_{1}$ and $W_{2}$. Since the outer conormal vector fields to $\bar{D}_{1}$ and $\bar{D}_{2}$ along their boundary have opposite directions, the difference of heights between $\tilde{\gamma}(a)$ and $\tilde{\gamma}(b)$ when we consider them in $W_{1}$ or $W_{2}$ is equal to $|T|$, but they have opposite signs (see 


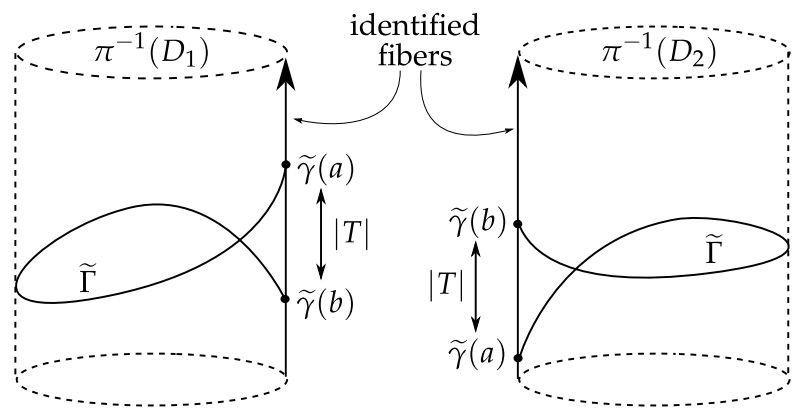

Figure 2. The curve $\widetilde{\Gamma}$ is represented in the solid cylinders $W_{1}$ and $W_{2}$ covering $\pi^{-1}\left(D_{1}\right)$ and $\pi^{-1}\left(D_{2}\right)$, respectively, and its endpoints lie on the vertical geodesic containing the vertical arrow representing a global vertical Killing vector field. After gluing along this geodesic, we conclude that the length of the fibers is an integer divisor of $|2 T|$.

the proof of Proposition 3.3). In other words, we will arrive at $\tilde{\gamma}(b)$ after traveling vertically from $\tilde{\gamma}(a)$ a distance of $|T|$, and, if we continue from $\tilde{\gamma}(b)$, we will arrive again at $\tilde{\gamma}(a)$ after the same distance (see Figure 2). Thus, the length of the fibers is an integer divisor of $|2 T|$. In particular, $\pi^{-1}\left(\bar{D}_{1}\right)$ and $\pi^{-1}\left(\bar{D}_{2}\right)$ are solid tori.

Now, observe that the curve $\widetilde{\Gamma}$ determines how $\pi^{-1}\left(\bar{D}_{1}\right)$ and $\pi^{-1}\left(\bar{D}_{2}\right)$ must be glued together, and $\widetilde{\Gamma}$ turns $n$ times in the vertical direction, so we can work in a $n$-sheet vertical covering space of both tori where $\widetilde{\Gamma}$ will look like Figure 2 after identifying the top and bottom faces of the cylinders. This way of gluing the two tori along $\Gamma$ provides a manifold diffeomorphic to $\mathbb{S}^{3}$, and the induced fibration is the Hopf fibration (see [Saveliev 1999]). By pulling the metric in $\mathbb{E}$ back via this diffeomorphism, (a) in the statement follows. Item (b) is also proved since we only need to undo the covering space procedure by taking a quotient with respect to a vertical translation of length $|2 T| / n$.

We can now combine the local existence given by Theorem 4.2 with Propositions 4.5 and 4.9 to obtain a description of all Killing submersions over a Riemannian 2-sphere.

Theorem 4.10. Let $g$ be a Riemannian metric on $\mathbb{S}^{2}$ and $\tau \in \mathscr{C}^{\infty}\left(\mathbb{S}^{2}\right)$. Up to isomorphism, there exists a unique Killing submersion over $\left(\mathbb{S}^{2}, g\right)$ with bundle curvature $\tau$ and whose total space is simply connected.

Proof. The uniqueness is a consequence of Theorem 2.8 and the description of the length of the fibers in Propositions 4.5 and 4.9. We will now assume that $T=\int_{\left(\aleph^{2}, g\right)} \tau \neq 0$ (the case $T=0$ is similar) and prove its existence. 
Consider an equator $\Gamma \subset \mathbb{S}^{2}$ splitting $\mathbb{S}^{2}$ in two hemispheres $D_{1}$ and $D_{2}$. By applying Theorem 4.2 in a neighborhood of $\bar{D}_{1}$ and $\bar{D}_{2}$, we obtain Killing submersions $\pi_{1}$ and $\pi_{2}$ over such neighborhoods with the desired bundle curvature and noncompact fibers. The argument in Proposition 4.9 guarantees that, after taking the quotient by vertical translations of length $|2 T|$, the two submersions can be glued together along $\pi^{-1}(\Gamma)$ to produce a (continuous) submersion $\pi: \mathbb{S}^{3} \rightarrow \mathbb{S}^{2}$. In order to prove that $\pi$ is smooth along $\pi^{-1}(\Gamma)$, observe that both $\pi_{1}$ and $\pi_{2}$ are defined in a neighborhood of $\Gamma$ where they share the same bundle curvature. Thus they locally coincide by Theorem 2.8 in a neighborhood of each $p \in \pi^{-1}(\Gamma)$.

In the previous section, we showed a constructive method to obtain trivial Killing submersions in a global way. Now, we will do the same for Killing submersions with $T \neq 0$ for round spheres $\mathbb{S}^{2}(\kappa)$ as base surfaces, though the method can be also adapted to the case $T=0$.

Let us consider the Hopf fibration given by (4-4) and the global frame in $\mathbb{S}^{3} \subset \mathbb{C}^{2}$ defined by

$$
\left(E_{1}\right)_{(z, w)}=(-\bar{w}, \bar{z}), \quad\left(E_{2}\right)_{(z, w)}=(-i \bar{w}, i \bar{z}), \quad\left(E_{3}\right)_{(z, w)}=(i z, i w) .
$$

This frame is orthonormal when we endow $\mathbb{S}^{3}$ with the round metric of curvature one. Let $\tau \in \mathscr{C}^{\infty}\left(\mathbb{S}^{2}(\kappa)\right)$ be a function with integral $T \neq 0$. Note that $\tau$ induces a function in $\tilde{\tau} \in \mathscr{C}^{\infty}\left(\mathbb{R}^{2}\right)$ via the stereographic projection given by (4-3). Theorem 4.2 allows us to construct a Killing submersion over $\mathbb{S}^{2}(\kappa) \backslash\{(0,0,1 / \sqrt{\kappa})\}$ with bundle curvature $\tilde{\tau}$. To do this, we calculate the associated function $\tilde{\eta} \in \mathscr{C}^{\infty}\left(\mathbb{R}^{2}\right)$ given by

$$
\tilde{\eta}(x, y)=2 \int_{0}^{1} \frac{s \cdot \tilde{\tau}(s x, s y)}{\left(1+(\kappa / 4) s^{2}\left(x^{2}+y^{2}\right)\right)^{2}} \mathrm{~d} s,
$$

which extends smoothly to infinity since $\tilde{\tau}$ extends smoothly to infinity, and thus induces $\eta \in \mathscr{C}^{\infty}\left(\mathbb{S}^{2}(\kappa)\right)$ by pulling back via the stereographic projection again. Hence this construction induces a Riemannian metric in $\mathbb{S}^{3}$ minus the fiber of $(0,0,1 / \sqrt{\kappa})$ but can be extended to the whole $\mathbb{S}^{3}$. It can be shown that this metric in $\mathbb{S}^{3}$ is the determined by the fact that

$$
\begin{aligned}
& Y_{1}=\frac{\sqrt{\kappa}}{2} E_{1}-\frac{\operatorname{Im}(z w)\left(\kappa T|w|^{2}-4 \pi \eta\left(\pi_{\mathrm{Hopf}}(z, w)\right)\right)}{2 \pi \sqrt{\kappa}|w|^{4}} E_{3}, \\
& Y_{2}=\frac{\sqrt{\kappa}}{2} E_{2}+\frac{\operatorname{Im}(z w)\left(\kappa T|w|^{2}-4 \pi \eta\left(\pi_{\mathrm{Hopf}}(z, w)\right)\right)}{2 \pi \sqrt{\kappa}|w|^{4}} E_{3}, \\
& Y_{3}=\frac{\pi}{T} E_{3},
\end{aligned}
$$

defines a global orthonormal frame. If $\tau$ is constant, then $\kappa T=4 \pi \tau$ and

$$
\eta\left(\pi_{\text {Hopf }}(z, w)\right)=|w|^{2} \tau,
$$


so the coefficients of $E_{3}$ in $Y_{1}$ and $Y_{2}$ vanish, and we get the metrics of the Berger spheres given by Torralbo [2012].

\section{Characterization of homogeneous Killing submersions}

Recall that a Riemannian manifold is said to be homogeneous when its isometry group acts transitively on the manifold. In this section, we will characterize the $\mathbb{E}(\kappa, \tau)$-spaces as the only simply connected homogeneous 3-manifolds admitting the structure of a Killing submersion.

In order to obtain this result, we will compute the Riemannian curvature of the total space $\mathbb{E}$ of a Killing submersion $\pi: \mathbb{E} \rightarrow M$ in terms of $M$ and the bundle curvature $\tau$. Since the computation is purely local, we will work in a canonical example (see Definition 2.6) associated to some functions $\lambda, a, b \in \mathscr{C}^{\infty}(\Omega)$ with $\lambda>0$ and $\Omega \subset \mathbb{R}^{2}$ (a different approach can be found in [Espinar and de Oliveira 2013]). The Koszul formula yields the Levi-Civita connection in the canonical orthonormal frame $\left\{E_{1}, E_{2}, E_{3}\right\}$ given by (2-3):

$$
\begin{array}{lll}
\bar{\nabla}_{E_{1}} E_{1}=-\frac{\lambda_{y}}{\lambda^{2}} E_{2}, & \bar{\nabla}_{E_{1}} E_{2}=\frac{\lambda y}{\lambda^{2}} E_{1}+\tau E_{3}, & \bar{\nabla}_{E_{1}} E_{3}=-\tau E_{2}, \\
\bar{\nabla}_{E_{2}} E_{1}=\frac{\lambda x}{\lambda^{2}} E_{2}-\tau E_{3}, & \bar{\nabla}_{E_{2}} E_{2}=-\frac{\lambda_{x}}{\lambda^{2}} E_{1}, & \bar{\nabla}_{E_{2}} E_{3}=\tau E_{1}, \\
\bar{\nabla}_{E_{3}} E_{1}=-\tau E_{2}, & \bar{\nabla}_{E_{3}} E_{2}=\tau E_{1}, & \bar{\nabla}_{E_{3}} E_{3}=0 .
\end{array}
$$

Since the Gaussian curvature $K_{M}$ of $M$ can be written in terms of the conformal factor as

$$
K_{M}=-\frac{\Delta_{0}(\log \lambda)}{\lambda^{2}}=\frac{\lambda_{x}^{2}+\lambda_{y}^{2}}{\lambda^{4}}-\frac{\lambda_{x x}+\lambda_{y y}}{\lambda^{3}},
$$

it is easy to work out any sectional curvature in $\mathbb{E}$.

Lemma 5.1. Let $\pi: \mathbb{E} \rightarrow M$ be a Killing submersion and $p \in \mathbb{E}$. Given a linear plane $\Pi \subseteq T_{p} \mathbb{E}$ with normal vector $N \in T_{p} \mathbb{E}$, its sectional curvature is

$$
K(\Pi)=v^{2}\left(K_{M}-3 \tau^{2}\right)+\left(1-v^{2}\right) \tau^{2}-2 v\left\langle N \wedge \xi_{p},(\bar{\nabla} \tau)_{p}\right\rangle,
$$

where $v=\left\langle N, \xi_{p}\right\rangle$, $\xi$ denotes the unit Killing vector field, $K_{M}$ is the Gaussian curvature of $M$ at $\pi(p)$, and $\tau$ is the bundle curvature at $p$.

The sectional curvature is $K_{M}-3 \tau^{2}$ for horizontal planes (i.e., planes which are orthogonal to $\xi$ ) and $\tau^{2}$ for vertical planes (i.e., planes containing the direction $\xi)$. In particular, we deduce that hyperbolic 3 -space, $\mathbb{H}^{3}$, does not admit a Killing submersion structure since $\mathbb{H}^{3}$ has constant sectional curvature of -1 and vertical planes in a Killing submersion always have nonnegative sectional curvature. 
On the other hand, given $v \in T_{p} \mathbb{E}$ with $\|v\|=1$, the Ricci curvature of $v$ can be easily deduced from Lemma 5.1 as

$$
\operatorname{Ric}(v)=\left(K_{M}-2 \tau^{2}\right)-\left\langle v, \xi_{p}\right\rangle^{2}\left(K_{M}-4 \tau^{2}\right)+2\left\langle v, \xi_{p}\right\rangle\left\langle v \wedge \xi_{p}, \bar{\nabla} \tau\right\rangle .
$$

The scalar curvature is $\rho=2\left(K_{M}-\tau^{2}\right)$.

Theorem 5.2. Let $\pi: \mathbb{E} \rightarrow M$ be a Killing submersion. If $\mathbb{E}$ is homogeneous, then both the Gaussian curvature of $M$ and the bundle curvature are constant. In particular, $\mathbb{E}$ is a $\mathbb{E}(\kappa, \tau)$-space or its quotient by a vertical translation.

Proof. Given $p \in \mathbb{E}$ and $v \in T_{p} \mathbb{E}$ with $\|v\|=1$, we can decompose $v=u+\sigma \xi_{p}$, where $u$ is horizontal and $\sigma \in \mathbb{R}$. From (5-2), we get

$$
\operatorname{Ric}(v)=\left(K_{M}-2 \tau^{2}\right)+\sigma \cdot\left(\left\langle u \wedge \xi_{p},(\bar{\nabla} \tau)_{p}\right\rangle-\left(K_{M}-4 \tau^{2}\right) \sigma\right) .
$$

Let $U_{p}=\left\{v \in T_{p} \mathbb{E}:\|v\|=1\right\}$ and $A_{p}=\left\{v \in U_{p}: \operatorname{Ric}(v)=K_{M}-2 \tau^{2}\right\}$. Observe that the vectors $v \in U_{p}$ satisfying $\sigma=0$ form a great circle and the same happens for $\left\langle u \wedge \xi_{p},(\bar{\nabla} \tau)_{p}\right\rangle-\left(K_{M}-4 \tau^{2}\right) \sigma=0$ if $(\bar{\nabla} \tau)_{p} \neq 0$ or $K_{M} \neq 4 \tau^{2}$. We deduce

$$
A_{p}= \begin{cases}U_{p} & \text { if } K_{M}=4 \tau^{2} \text { and }(\bar{\nabla} \tau)_{p}=0, \\ \text { a great circle } & \text { if } K_{M} \neq 4 \tau^{2} \text { and }(\bar{\nabla} \tau)_{p}=0, \\ \text { two great circles } & \text { if }(\bar{\nabla} \tau)_{p} \neq 0\end{cases}
$$

Let $f: \mathbb{E} \rightarrow \mathbb{E}$ be an isometry. Since any two great circles in a sphere intersect and $\mathrm{d} f_{p}$ maps great circles in $U_{p}$ to great circles in $U_{f(p)}$, we deduce that $\mathrm{d} f_{p}\left(A_{p}\right)$ and $A_{f(p)}$ intersect. As a consequence, $K_{M}-2 \tau^{2}$ attains the same value at the points $p$ and $f(p)$. If $\mathbb{E}$ is homogeneous, then this implies that $K_{M}-2 \tau^{2}$ is constant, but on the other hand, the scalar curvature $2\left(K_{M}-\tau^{2}\right)$ is also constant; hence both $K_{M}$ and $\tau$ are constant.

Remark 5.3. Given a 3-dimensional metric Lie group $G$ (i.e., it is endowed with a left-invariant metric) with isometry group of dimension 3 , it is homogeneous. We deduce that the set of points where a Killing vector field (i.e., a right-invariant vector field) is unitary has empty interior. Otherwise, this open subset would be locally isometric to a $\mathbb{E}(\kappa, \tau)$-space, and this is impossible (see [Meeks and Pérez 2012] for a detailed description of metric Lie groups).

Finally, let us mention that the condition $K_{M}=4 \tau^{2}$ does not imply that $\mathbb{E}$ has constant sectional curvature (unless $\tau$ is constant), but it says that horizontal and vertical planes have the same sectional curvature. Note that, if $(\nabla \tau)_{p} \neq 0$ and $K_{M}=4 \tau^{2}$ at some $p \in M$, then the set $A_{p}$ in (5-3) consists of two orthogonal great circles in the unit sphere $U_{p}$. 


\section{Acknowledgements}

I would like to express my gratitude to Hojoo Lee, Pablo Mira and Joaquín Pérez for suggesting some improvements in the preparation of this paper. I would also like to thank Luis Guijarro and Carlos Ivorra for showing me some useful references.

\section{References}

[Abresch and Rosenberg 2005] U. Abresch and H. Rosenberg, "Generalized Hopf differentials", Mat. Contemp. 28 (2005), 1-28. MR 2006h:53004 Zbl 1118.53036

[Besse 2008] A. L. Besse, Einstein manifolds, Springer, Berlin, 2008. MR 2008k:53084 Zbl 1147.53001

[do Carmo 1992] M. P. do Carmo, Riemannian geometry, Birkhäuser, Boston, 1992. MR 92i:53001 Zbl 0752.53001

[Dajczer and de Lira 2009] M. Dajczer and J. H. de Lira, "Killing graphs with prescribed mean curvature and Riemannian submersions", Ann. Inst. H. Poincaré Anal. Non Linéaire 26:3 (2009), 763-775. MR 2010f:53093 Zbl 1169.53046

[Dajczer and de Lira 2012] M. Dajczer and J. H. de Lira, "Conformal Killing graphs with prescribed mean curvature", J. Geom. Anal. 22:3 (2012), 780-799. MR 2927678 Zbl 1261.53058

[Daniel 2007] B. Daniel, "Isometric immersions into 3-dimensional homogeneous manifolds", Comment. Math. Helv. 82:1 (2007), 87-131. MR 2008a:53058 Zbl 1123.53029

[Daniel et al. 2009] B. Daniel, L. Hauswirth, and P. Mira, "Homogeneous 3-manifolds", Lecture notes from the 4th KIAS Workshop on Differential Geometry, Korea Institute for Advanced Study, Seoul, 2009.

[Espinar and de Oliveira 2013] J. M. Espinar and I. S. de Oliveira, "Locally convex surfaces immersed in a Killing submersion”, Bull. Braz. Math. Soc. (N.S.) 44:1 (2013), 155-171. MR 3077638 Zbl 1270.53080

[Greub et al. 1976] W. Greub, S. Halperin, and R. Vanstone, Connections, curvature, and cohomology, III: Cohomology of principal bundles and homogeneous spaces, Pure and Applied Mathematics 47, Academic Press, New York, 1976. MR 53 \#4110 Zbl 0372.57001

[Javaloyes et al. 2013] M. A. Javaloyes, L. Lichtenfelz, and P. Piccione, "Almost isometries of non-reversible metrics with applications to stationary spacetimes", preprint, 2013. arXiv 1205.4539

[Jost 2002] J. Jost, Compact Riemann surfaces: an introduction to contemporary mathematics, 2nd ed., Springer, Berlin, 2002. MR 2003h:32020 Zbl 1086.30038

[Leandro and Rosenberg 2009] C. Leandro and H. Rosenberg, "Removable singularities for sections of Riemannian submersions of prescribed mean curvature", Bull. Sci. Math. 133:4 (2009), 445-452. MR 2011a:53105 Zbl 1172.53038

[Lee and Manzano 2013] H. Lee and J. M. Manzano, "Generalized Calabi's correspondence and complete spacelike surfaces", preprint, 2013. arXiv 1301.7241

[Manzano and Torralbo 2012] J. M. Manzano and F. Torralbo, "New examples of constant mean curvature surfaces in $\mathbb{S}^{2} \times \mathbb{R}$ and $\mathbb{U}^{2} \times \mathbb{R}$ ”, preprint, 2012. To appear in Michigan Math. J. arXiv 1104.1259

[Meeks and Pérez 2012] W. H. Meeks, III and J. Pérez, "Constant mean curvature surfaces in metric Lie groups", pp. 25-110 in Geometric analysis: partial differential equations and surfaces (Granada, 2010), edited by J. Pérez and J. A. Gálvez, Contemp. Math. 570, Amer. Math. Soc., Providence, RI, 2012. MR 2963596 Zbl 1267.53006 
[Meroño and Ortiz 2014] M. A. Meroño and I. Ortiz, "First stability eigenvalue characterization of CMC Hopf tori into Riemannian Killing submersions”, J. Math. Anal. Appl. 417:1 (2014), 400-410. MR 3191435

[Nash 1956] J. Nash, "The imbedding problem for Riemannian manifolds", Ann. of Math. (2) 63 (1956), 20-63. MR 17,782b Zbl 0070.38603

[Rosenberg et al. 2010] H. Rosenberg, R. Souam, and E. Toubiana, "General curvature estimates for stable $H$-surfaces in 3-manifolds and applications", J. Differential Geom. 84:3 (2010), 623-648. MR 2011g:53015 Zbl 1198.53062

[Saveliev 1999] N. Saveliev, Lectures on the topology of 3-manifolds: an introduction to the Casson invariant, de Gruyter, Berlin, 1999. MR 2001h:57024 Zbl 0932.57001

[Souam and Van der Veken 2012] R. Souam and J. Van der Veken, "Totally umbilical hypersurfaces of manifolds admitting a unit Killing field”, Trans. Amer. Math. Soc. 364:7 (2012), 3609-3626. MR 2901226 Zbl 1277.53021

[Steenrod 1951] N. Steenrod, The topology of fibre bundles, Princeton Mathematical Series 14, Princeton University Press, Princeton, NJ, 1951. MR 12,522b Zbl 0054.07103

[Torralbo 2012] F. Torralbo, "Compact minimal surfaces in the Berger spheres", Ann. Global Anal. Geom. 41:4 (2012), 391-405. MR 2897028 Zbl 1242.53076

Received June 6, 2013.

José M. MANZANO

DEPARTAMENTO DE GEOMETRÍA Y TOPOLOGÍA

UNIVERSIDAD DE GRANADA

18071 GRANADA

SPAIN

jmmanzano@ugr.es 


\title{
PACIFIC JOURNAL OF MATHEMATICS
}

\author{
msp.org/pjm
}

Founded in 1951 by E. F. Beckenbach (1906-1982) and F. Wolf (1904-1989)

\section{EDITORS}

Don Blasius (Managing Editor)

Department of Mathematics

University of California

Los Angeles, CA 90095-1555

blasius@math.ucla.edu

\author{
Paul Balmer \\ Department of Mathematics \\ University of California \\ Los Angeles, CA 90095-1555 \\ balmer@math.ucla.edu \\ Robert Finn \\ Department of Mathematics \\ Stanford University \\ Stanford, CA 94305-2125 \\ finn@math.stanford.edu \\ Sorin Popa \\ Department of Mathematics \\ University of California \\ Los Angeles, CA 90095-1555 \\ popa@math.ucla.edu
}

\author{
Vyjayanthi Chari \\ Department of Mathematics \\ University of California \\ Riverside, CA 92521-0135 \\ chari@math.ucr.edu \\ Kefeng Liu \\ Department of Mathematics \\ University of California \\ Los Angeles, CA 90095-1555 \\ liu@math.ucla.edu \\ Jie Qing \\ Department of Mathematics \\ University of California \\ Santa Cruz, CA 95064 \\ qing@ cats.ucsc.edu
}

\section{PRODUCTION}

Silvio Levy, Scientific Editor, production@msp.org

\section{SUPPORTING INSTITUTIONS}

ACADEMIA SINICA, TAIPEI

CALIFORNIA INST. OF TECHNOLOGY

INST. DE MATEMÁTICA PURA E APLICADA

KEIO UNIVERSITY

MATH. SCIENCES RESEARCH INSTITUTE

NEW MEXICO STATE UNIV.

OREGON STATE UNIV.

\author{
STANFORD UNIVERSITY \\ UNIV. OF BRITISH COLUMBIA \\ UNIV. OF CALIFORNIA, BERKELEY \\ UNIV. OF CALIFORNIA, DAVIS \\ UNIV. OF CALIFORNIA, LOS ANGELES \\ UNIV. OF CALIFORNIA, RIVERSIDE \\ UNIV. OF CALIFORNIA, SAN DIEGO \\ UNIV. OF CALIF., SANTA BARBARA
}

\author{
Daryl Cooper \\ Department of Mathematics \\ University of California \\ Santa Barbara, CA 93106-3080 \\ cooper@math.ucsb.edu \\ Jiang-Hua Lu \\ Department of Mathematics \\ The University of Hong Kong \\ Pokfulam Rd., Hong Kong \\ jhlu@maths.hku.hk \\ Paul Yang \\ Department of Mathematics \\ Princeton University \\ Princeton NJ 08544-1000 \\ yang@math.princeton.edu
}

These supporting institutions contribute to the cost of publication of this Journal, but they are not owners or publishers and have no responsibility for its contents or policies.

See inside back cover or msp.org/pjm for submission instructions.

The subscription price for 2014 is US $\$ 410 /$ year for the electronic version, and \$535/year for print and electronic.

Subscriptions, requests for back issues and changes of subscribers address should be sent to Pacific Journal of Mathematics, P.O. Box 4163, Berkeley, CA 94704-0163, U.S.A. The Pacific Journal of Mathematics is indexed by Mathematical Reviews, Zentralblatt MATH, PASCAL CNRS Index, Referativnyi Zhurnal, Current Mathematical Publications and Web of Knowledge (Science Citation Index).

The Pacific Journal of Mathematics (ISSN 0030-8730) at the University of California, c/o Department of Mathematics, 798 Evans Hall \#3840, Berkeley, CA 94720-3840, is published twelve times a year. Periodical rate postage paid at Berkeley, CA 94704, and additional mailing offices. POSTMASTER: send address changes to Pacific Journal of Mathematics, P.O. Box 4163, Berkeley, CA 94704-0163.

PJM peer review and production are managed by EditFLOW ${ }^{\circledR}$ from Mathematical Sciences Publishers.

\section{PUBLISHED BY}

\section{mathematical sciences publishers \\ nonprofit scientific publishing}

http://msp.org/

(C) 2014 Mathematical Sciences Publishers 


\section{PACIFIC JOURNAL OF MATHEMATICS}

Volume $270 \quad$ No. $2 \quad$ August 2014

Disjointification inequalities in symmetric quasi-Banach spaces and 257 their applications

SERgey Astashinin, Fedor A. SukocheV and DMitriy

ZANIN

Hamiltonian evolutions of twisted polygons in parabolic manifolds:

287

The Lagrangian Grassmannian

GLORIA MARÍ BEFFA

On Schwarz-Christoffel mappings

MARTIN CHUAQUi and CHRISTIAN POMMERENKE

Vanishing viscosity in the plane for nondecaying velocity and vorticity, II

ELAINE COZZI

Affine quantum Schur algebras and affine Hecke algebras

QIANG FU

On the classification of Killing submersions and their isometries

JosÉ M. MANZANO

Locally Lipschitz contractibility of Alexandrov spaces and its applications

Ayato Mitsuishi and TAKaO Yamaguchi

Sequences of open Riemannian manifolds with boundary

RAQUEL PERALES and CHRISTINA SORMANI

Invariant differential operators on a class of multiplicity-free spaces 